\title{
Understanding the Effects of Stimulant Medications on Cognition in Individuals with Attention-Deficit Hyperactivity Disorder: A Decade of Progress
}

\author{
James Swanson ${ }^{1}$, Ruben D Baler ${ }^{2}$ and Nora D Volkow*,2 \\ ${ }^{1}$ Department of Pediatrics, University of California, Irvine, CA, USA; ${ }^{2}$ National Institute on Drug Abuse, National Institutes of \\ Health, Bethesda, MD, USA
}

\begin{abstract}
The use of stimulant drugs for the treatment of children with attention-deficit hyperactivity disorder (ADHD) is one of the most widespread pharmacological interventions in child psychiatry and behavioral pediatrics. This treatment is well grounded on controlled studies showing efficacy of low oral doses of methylphenidate and amphetamine in reducing the behavioral symptoms of the disorder as reported by parents and teachers, both for the cognitive (inattention and impulsivity) and noncognitive (hyperactivity) domains. Our main aim is to review the objectively measured cognitive effects that accompany the subjectively assessed clinical responses to stimulant medications. Recently, methods from the cognitive neurosciences have been used to provide information about brain processes that underlie the cognitive deficits of ADHD and the cognitive effects of stimulant medications. We will review some key findings from the recent literature, and then offer interpretations of the progress that has been made over the past decade in understanding the cognitive effects of stimulant medication on individuals with ADHD.

Neuropsychopharmacology Reviews (2011) 36, 207-226; doi: I 0. 1038/npp.20 I0.160; published online 29 September 2010
\end{abstract}

Keywords: methylphenidate; amphetamine; dopamine; prefrontal cortex; executive function; cognitive enhancement

\section{INTRODUCTION}

Cognitive deficits and behavioral symptoms associated with attention-deficit/hyperactivity disorder (ADHD) have been studied intensively and are well documented in the literature. Stimulant drugs (methylphenidate (MP) and amphetamine (AMP)) are particularly effective for the clinical treatment of ADHD, and accordingly, pediatricians and psychiatrists have been prescribing them for over seven decades (Bradley, 1937). Clear reductions in symptoms justify treatment with stimulant medications (MTA, 1999b), but the cognitive effects are less clear. Here, we will review the current understanding of some underlying neural processes that may account for the ADHD symptoms of inattention and impulsivity, and we will review evidence from neuropsychological and neuroimaging studies about how stimulant medications may affect these processes. To focus our review, we will not revisit related topics that

${ }^{*}$ Correspondence: Dr ND Volkow, National Institute on Drug Abuse, National Institutes of Health, Bethesda, MD 20892, USA, Tel: +1301443 6480, Fax: + 1301443 9127, E-mail: nvolkow@nida.nih.gov

Received 17 May 2010; revised 8 August 2010; accepted 9 August 2010 are addressed elsewhere: the medical use of non-stimulant drugs to treat ADHD (Dopheide and Pliszka, 2009; Waxmonsky, 2005), the non-medical use of stimulants and non-stimulants for cognitive enhancement (Sahakian and Morein-Zamir, 2007; Stix, 2009), and nonmedical alternative treatments based on neurofeedback (Gevensleben et al, 2009) and working memory training (Klingberg et al, 2005) that have recently been evaluated and have gained some support in randomized clinical trials.

In the Diagnostic and Statistical Manual (DSM) of the American Psychiatric Association, Version IV (APA, 1994, 2000), the cognitive symptoms of ADHD are grouped into two domains: inattention and hyperactivity/impulsivity. Currently, nine are specified for inattention (poor attending to details, sustaining attention, listening, organizing and finishing tasks, exerting mental effort, ignoring extraneous, and remembering things and activities), but only three for impulsivity (blurting out answers, cannot wait, and interrupting others), which are grouped with six motor symptoms of hyperactivity (often fidgeting, leaving seat in the classroom, running about, not able to play quietly, 'on the go', and talking excessively). 
These cognitive symptoms have been grouped, ungrouped, and re-grouped in different ways in the serial revisions of the DSM (DSM III, 1980; DSM III-R, 1987; and DSM IV, 1994); hence a variety of subtypes have been proposed and evaluated (respectively, ADD with or without hyperactivity; ADHD without subtypes; ADHD predominately inattentive, predominately hyperactive/impulsive, or combined types). This vacillation suggests great difficulty in understanding the place for cognitive deficits in the clinical diagnosis of ADHD. This may be related to the complexity of definitions of the same domains in the cognitive neurosciences. For example, Winstanley et al (2006) described two general types of impulsivity-impulsive choice and impulsive action - and (Posner and Rothbart, 2007) described three general components of attention-alerting, orienting, and executive control. More precise definition and measurement of cognitive deficits related to inattention and impulsivity may help advance the understanding of cognitive deficits in ADHD.

Over the past decade, specific laboratory tasks have been used for this purpose. Here, we will focus on three of the most prominent that use reaction time (RT) as a measure of performance: (a) the Stop Signal Task (STOP) that requires the cued inhibition of response to stimulus after a signal; (b) the reverse continuous performance task (CPT) (GoNoGo) that requires response to most but not all stimuli in a series; and (c) the attentional network task (ANT) that requires response to central stimuli in the face of temporal, spatial, and conflicting surrounding cues. We will build on reviews that relate these tasks to the brain imaging literature of structure (Krain and Castellanos, 2006), function (Dickstein et al, 2006), and connectivity (Bush, 2010), and emphasize advances over the past decade in the conceptualization and evaluation of neural processes and networks that may be abnormal in ADHD, and thus may mediate the effects of stimulant medication on cognition in individuals with ADHD.

\section{BACKGROUND STUDIES ON THE EFFECTS OF STIMULANTS IN COGNITION IN ADHD}

\section{Cognitive Deficits}

One purpose of clinical treatment is to correct a deficit; this is important to consider in the assessment of cognitive effects of stimulant drugs in light of the current controversy about non-medical use of stimulants and cognitive enhancement (Sahakian and Morein-Zamir, 2007; Stix, 2009). The Yerkes-Dodson Law provides a theoretical framework for this (Diamond et al, 2007; Arnsten, 2009). At some doses and on some tasks (Dodds et al, 2008), stimulant drugs may have the same direction of effect (cognitive enhancement) in some individuals without ADHD (Clatworthy et al, 2009) as well as in those with ADHD. The non-medical use of stimulant drugs for enhancement rather than the medical use for correction of deficit defines an ethical issue addressed elsewhere (Sahakian and Morein-Zamir, 2007;
Swanson et al, in press). In this section, we will focus on definitions of cognitive deficits associated with diagnoses of ADHD in children.

A brief history and succinct review of theories of attention in ADHD will set the stage for our review of the recent literature. Sergeant and Scholten (1983) applied a cognitive energetic theory of information processing and found that in ADHD children central processing stage was intact and that deficits are restricted to motor organization and output stages of information processing. Swanson et al (1991b) applied a cognitive anatomical theory of attention (Posner and Petersen, 1990), and found that in ADHD children, the posterior system for engaging attention was intact and that deficits were restricted to the anterior system of maintaining (disengaging and moving) attention. Pennington and Ozonoff (1996) proposed that the core deficits of ADHD were linked to the abnormal development of executive functions in childhood. The most prominent and influential theory (Barkley, 1997) was based on the Fuster theory of frontal lobe function (Fuster, 1980), and it proposed that ADHD was characterized by a core deficit in response inhibition, which theoretically would lead to cognitive symptoms as secondary manifestations.

The core deficit theories of ADHD were assessed in comprehensive reviews by Nigg (2005) and Willcutt et al (2005). Both used the concept of 'effect size' (es) (defined as the difference between an ADHD and control group, expressed in standard deviation units) to compare cognitive deficits across a variety of neuropsychological tasks. In Table 1, we present their lists of tasks that provide an empirical basis to define cognitive deficits and the relative es. These reviews mark a watershed point in the literature on cognitive deficits of ADHD: they pointed out that few children with ADHD showed pervasive deficits across tests, concluded that executive function deficits were not necessary and sufficient causes of ADHD, and contributed to the shift from core deficit to multiple deficit theories.

Laboratory measures of inattention and impulsivity have been used to assess the pervasive cognitive deficits manifested by children with ADHD across multiple domains. In some studies, multiple tests of executive function and motor inhibition were used (Oosterlaan et al, 2005; Scheres et al, 2004). In others, standard batteries of neuropsychological tests were used: two prime examples are the Cambridge Neuropsychological Test Automated Battery (CANTAB) (Rhodes et al, 2005, 2006) and the Maudsley Attention and Response Suppression (MARS) (Rubia et al, 2007). This approach has uncovered ADHD-control group differences (deficits) on tests of temporal and parietal lobe function (spatial recognition and span, pattern recognition, and delayed matching to sample), as well as frontal lobe function (working memory, planning and strategy formation, and setshifting). Another approach has been to use a specific task to assess cognitive components of inattention and impulsivity in ADHD children, and over the past decade three have emerged as the most prominent in the literature: the Go-NoGo task, the STOP task, and the ANT. 
TABLE 1 Tasks Reviewed by Nigg (2005) and Willcutt et al (2005)

\begin{tabular}{|c|c|c|c|}
\hline Nigg & & Willcutt & \\
\hline Spatial working memory & 1.14 to 0.75 & Spatial working memory & 0.63 \\
\hline Response suppression (Stop Task) & 0.94 to 0.61 & Stop task & 0.61 \\
\hline \multirow[t]{2}{*}{ Signal detection (CPT) } & 0.72 & CPT omissions & 0.64 \\
\hline & & CPT commissions & 0.51 \\
\hline Stroop naming speed & 0.69 & & \\
\hline Full scale IQ & 0.61 & & \\
\hline Set shifting (Trails B) & 0.55 to 0.75 & Trails B & 0.55 \\
\hline \multirow[t]{2}{*}{ Planning (Tower tasks) } & 0.51 to 0.69 & Tower of London & 0.51 \\
\hline & & Tower of Hanoi & 0.69 \\
\hline Mazes & 0.58 & Porteus Mazes & 0.58 \\
\hline Verbal working memory & 0.51 to 0.41 & Verbal working memory & 0.55 \\
\hline Fluency & 0.27 & & \\
\hline Decision speed (Go Task) & 0.49 & & \\
\hline \multirow[t]{2}{*}{ WCST perseverations } & 0.36 to 0.53 & WCST perseverations & 0.46 \\
\hline & & R-O copying (Organzation) & 0.43 \\
\hline Stroop interference & 0.25 & & \\
\hline Covert VP orienting & 0.20 & & \\
\hline
\end{tabular}

Numbers represent effect sizes, which are defined as the difference between an ADHD and control group, and expressed in standard deviation units.

A typical version of the Go-NoGo task requires rapid responding (eg, with a key press) to a series of 'Go' stimuli (eg, letters) and selective inhibition of the motor response to infrequent 'NoGo' stimuli (eg, specific letters). The Conners version of the CPT (Conners et al, 2003) required a key press response to all but one letter (eg, all but $\mathrm{X}$ ), and in a study to develop population norms (Epstein et al, 2003), about $20 \%$ of the commission errors occurred owing to failure to withhold a response. Comparisons of Go-NoGo performance of ADHD and control children revealed significant differences in commission errors, as well as variability of RTs, omission errors, and a derived measure of perceptual sensitivity $\left(d^{\prime}\right)$ based on signal detection theory. These performance deficits were associated with differences in brain activation as assessed by fMRI (Dickstein et al, 2006). During the performance of a GoNoGo task, in the ADHD compared with controls, some important findings were reduced activation of right caudate nucleus, but increased activation in the right inferior parietal lobe and posterior cingulate (Durston et al, 2003); increased activation of the posterior cingulate and dorsolateral prefrontal and parietal brain regions (Schulz et al, 2004; Tamm et al, 2004); attenuated activity in frontostriatal regions when tested off medication, which were increased when tested on medication along with increases in cerebellar regions (Epstein et al, 2006); and disrupted structural connectivity within frontostriatal networks (Casey et al, 2007). These Go-NoGo fMRI studies reveal hypoactivation involving relatively smaller regions within the inferior frontal cortex, anterior cingulate cortex, and precentral gyrus, as well as hyperactivation in medial frontal gyrus and right paracentral lobule. Reviews of the literature (Dickstein et al, 2006; Fassbender and Schweitzer, 2006) concluded that ADHD children may rely on brain functions based on visual and motor processing of information associated with performance strategies that allow them to compensate for deficits in executive functions.

The STOP task requires the inhibition of the Go response (eg, a key press to visual stimuli) when a Stop signal (eg, a tone) occurs. One version had fixed delays between the Stop and Go signals, which may result in a strategyrelated delay in response to Go signals to avoid unsuccessful inhibitions to the Stop signal. To prevent this, another version incorporates a dynamic tracking adjustment of the delay between Go and Stop signals, decreasing it after successful and increasing it after unsuccessful trials to elicit about $50 \%$ successful inhibitions to the Stop signal. On the basis of an underlying model of the speed of the two mental processes, the RT to the Stop signal is estimated indirectly. The STOP task has been used in many studies of ADHD children to evaluate inhibition deficits (see reviews by Lijffijt et al (2005), Oosterlaan et al (1998), and Willcutt et al (2005)). A recent review (Alderson et al, 2007) contrasted the es for the Stop-signal reaction time (SSRT, es $=0.63$ ) and mean RT (MRT, es $=0.45)$, as well as on a measure of Stop Signal Delay (SSD) defined as the difference between them (ie, SSD $=$ MRT-SSRT, es $=-0.025$ ), and concluded that children with ADHD 
had slower and more variable RTs to primary stimuli (ie, go-stimuli) as well as a Stop signal, and thus they appeared to have '... an underlying attention deficit rather than deficient inhibitory control' ( $p$ 755). Studies that combined the STOP task with fMRI also showed differential activation patterns of a distributive nature, which do not support models that hinge on the dysfunction in any one frontal subregion (Dickstein et al, 2006). The interpretation of task-related group differences is complicated by a significant group difference in activity while performing the STOP task in the imaging setting. For example, Pliszka et al (2006) used a visual fixed interval STOP task and reported that more children with ADHD (15 of 32) than without (eight out of 23) were eliminated owing to motion artifacts, and significantly higher scores for allowable movement were present in the remaining censored group of ADHD children than in the control group (2.57 vs 1.71).

The ANT was developed by Posner and his colleagues (Fan et al, 2002) as a simplified variant of the Posner visualspatial orienting task (Posner et al, 1988; Swanson et al, 1991b) to facilitate the evaluation of components of attention - alerting (elicited by a temporal cue), orienting (elicited by a spatial cue), and executive control (elicited by visual-spatial conflict). It is based on the Eriksen-Flanker Task, using a right or left pointing arrow (or swimming fish for children) surrounded by stimuli pointing in the same (congruent flankers) or the opposite direction (incongruent flankers) to establish cognitive conflict. The speed of each of the three component processes is estimated by differences in RTs for trials with different types of cues or flankers. The ANT has been included in the NIH Toolbox of cognitive tasks for the assessment of attention across the lifespan (Jin-Shei et al, 2010). It has been modified for the assessment of cognitive deficits associated with brain damage as a test for attentional performance (Drechsler et al, 2005). Various versions of the ANT have revealed deficits in ADHD vs control groups on measures of the executive function network, but the observed response patterns (slower RTs and more errors, particularly on conflict trials), did not reflect impulsive responding (Johnson et al, 2008; Konrad et al, 2006). Konrad et al (2006) also used fMRI to evaluate brain regions activated by trials requiring alerting (anterior cingulate gyrus activation was greater in the control than in the ADHD group, but the brainstem activation was greater in the ADHD than in the control group), orienting trials (putamen activation was greater in the ADHD than in the control group), and executive control (frontal activation was greater in the control than in the ADHD group, but brainstem and parietal activation was greater in the ADHD than in the control group). This pattern suggested that some individuals in the control group have the expected activation of the frontalstriatal circuitry, whereas the ADHD group manifest compensatory activation of different brain regions.

In summary, we should emphasize that over the past decade, there has been a shift in the consensus view of cognitive deficits associated with ADHD. Earlier theories had proposed core deficits in the frontal lobe function, but a variety of studies favored multicomponent theories based on various cognitive deficits formulated to account for the observed heterogeneity of cognitive impairments manifested in the clinical samples of ADHD cases. Some fMRI studies of ADHD confirmed a wide range of brain regions with the evidence of deficits (hypoactivation relative to controls), but others suggest that ADHD children may compensate for cognitive deficits by using alternative neural processes to perform these tasks compared with control children, which may produce patterns of hyperactivation as well as hypoactivation in different brain regions.

\section{Cognitive Effects of Stimulants}

The evaluation of cognitive effects of stimulants on children with ADHD has a long history (Knights, 1974). The conclusions have become more consistent over time as the literature on this topic increased exponentially (Conners, 2002; Ottenbacher and Cooper, 1983; Rapport and Kelley, 1993; Swanson et al, 1991a). A basic finding is that the percentage of children who benefit depends on the task used to assess improvement, with the highest response rates for the assessment of activity (with decreases considered beneficial) and the lowest response rates for the assessment of learning or problem solving (with increases considered beneficial). An early study of dose-related effects (Sprague and Sleator, 1977) had suggested that the optimal dose for cognitive effects was lower than that for behavioral effects of $M P$, and in an early study of the effects in non-ADHD individuals, Rapoport et al (1978) suggested that the response in ADHD individuals was not 'paradoxical', but instead was in the same direction for some measures in control (non-ADHD) individuals. Sahakian and Robbins (1977) and Robbins and Sahakian (1979) suggested that this task-dependent pattern of response may be a consequence of the general dose-related effect of stimulants to increase stereotypic behavior, which would improve performance on some tasks (eg, tasks that require sustained attention for repetitive action and thought), but impair it on others (eg, tasks that require reversals in cognitive strategy). Pietrzak et al (2006) provides a meta-analysis of the recent literature on placebo-controlled studies of the effects of MP on a variety of neuropsychological tasks, and in studies comparing effects for more than one clinical dose, higher doses produced greater improvements than lower doses for some tasks (attention, vigilance, memory, and working memory), but no additional improvements on others (planning, cognitive flexibility, inhibitory control, naming, and motor speed).

Dose-related effects of stimulants have been evaluated using batteries as well as specific tasks. For example, Coghill and his colleagues (Coghill et al, 2007; Rhodes et al, 2005, 2006) used the CANTAB to evaluate the acute and chronic effects of MP ( 0.3 and $0.6 \mathrm{mg} / \mathrm{kg}$ doses). In stimulant-naïve children with ADHD, controlling for practice effects in a randomized clinical trial (RCT) with between-subject 
comparisons (Rhodes et al, 2006), improvement was documented on some tasks without a major executive function (EF) component (complex reaction time, spatial recognition memory reaction time, and delayed matchingto-sample) but surprisingly not those designed to assess the executive function components of neuropsychological performance (inhibition, working memory, strategy formation, planning, and set-shifting). In the follow-up study of chronic (four-week) MP administration (Coghill et al, 2007), a significant behavioral response to medication compared with placebo was documented by parent and teacher ratings on the Conners' 10 -item rating scale in $59 \%$ of the children at some dose, but the effect of dose was not significant and the optimal dose varied across children (33\% when treated with a low- $0.3 \mathrm{mg} / \mathrm{kg}$ - dose and $43 \%$ when treated with the high- $0.6 \mathrm{mg} / \mathrm{kg}$ - dose). The chronic cognitive response to MP was shown on visual memory tests (pattern matching, delayed matching to sample, and spatial memory), but not on EF tests (except the Go-NoGo test). This well-designed and implemented study of the cognitive effects of stimulant medication did not confirm expectations that the response would correct EF deficits, but also did not document a dose-related impairment on any of the tasks in the battery.

Scheres et al (2003) provides an example of the use of a specific task (the STOP task) to evaluate dose-response to MP in a double-blind, placebo-controlled, crossover study of three doses $(5,10$, and $20 \mathrm{mg})$. The overall effect of medication was significant owing to faster SSRTs and lower RT variability, but the dose effect was not significant, suggesting that the maximum cognitive benefit may be elicited by a low dose of medication. Spencer et al (2009) evaluated the cognitive response of children with ADHD to stimulant medication based on an RT task of discriminating two stimuli ( $\mathrm{X}$ or $\mathrm{O}$ ) in a double-blind, dose-response, crossover study of placebo, low $(0.9 \mathrm{mg} / \mathrm{kg} /$ day $)$ and high (1.8 mg/kg/day) MP doses delivered by controlled-release formulations. Medication produced a significant reduction of the mode and SD from the mode (rather than the mean, which is correlated with SD) for the low as well as high doses, which was not owing to a speed-accuracy tradeoff, but instead reflected an overall increased efficiency of responding (faster and more accurate responses). The evaluation of the effects of dose on mode RT suggests that the full effect of cognitive enhancement was manifested at the low dose.

Overall, the literature suggests some general findings that have been consistent across time and studies. Stimulantrelated improvements in ADHD children have been documented across a wide range of cognitive functions. In well-controlled studies using batteries, stimulant-related cognitive enhancements were more prominent on tasks without an executive function component (complex reaction time, spatial recognition memory reaction time, and delayed matching-to-sample) than on tasks with an executive function component (inhibition, working memory, strategy formation, planning, and set-shifting). Doseresponse studies of stimulant medications suggest that the optimal dose varies across individuals and depends somewhat on the domain of function, with high doses tending to produce greater enhancement on some (eg, vigilance) but not others (eg, planning), without clear evidence of completely correcting cognitive deficits associated with ADHD.

\section{Long-term Cognitive Effects of Stimulant Medication}

Most studies of the cognitive effects of stimulant medication focused on acute effects, but recently a few follow-up studies have provided some data that have generated speculations about long-term effects. One of the most controversial (BBC, 2010) has been the Multimodal Treatment study of ADHD (MTA). The MTA evaluated a large sample $(n=579)$ in a 14-month RCT of groups assigned to intensive pharmacological treatment with stimulant medication (MedMgt), intensive non-pharmacological treatment with behavior modification (Beh), treatment with the combination of these two modalities of treatment (Comb), or to a treatment in the community for comparison to the treatments-by-protocol (CC). The acute effects of stimulant medication were documented in a double-blind, doseresponse titration trial to select optimal starting dose for each participant (MTA, 1999a). At the end of the 14-month treatment-by-protocol phase, the chronic effects of stimulant medication were evaluated by comparison of the treatments with (MedMgt and Comb) and without (Beh and CC) the MTA medication algorithm as a component of the assigned treatment. This RCT comparison documented the relative superiority of stimulant medication on the primary outcome measure (ie, parent and teacher ratings of symptom severity). In parallel with the behavioral ratings of ADHD symptoms, relative superiority of medication on cognitive outcomes evaluated by tests of achievement on reading and math were documented. The clinically optimal dose varied across individuals, but over the 14 months of treatment-by-protocol, increases in dose of about $20 \%$ were made to maintain full efficacy (Vitiello et al, 2001). The MTA was continued as a prospective observational study. In the naturalistic follow-up at 2, 3, 6, and 8 years, the natural history of medication use was clear: most children who were assigned to treatment with and were actually treated with stimulants did not continue this component of treatment. Current percentage of ADHD cases who were being treated with stimulant medications at the 8 year follow-up was $32.5 \%$. The follow-up assessments revealed that by the 3-year assessment point, the initial relative benefits of assignment to the medication conditions and of current medication use were no longer significant. This suggests that the relative benefits of childhood treatment with stimulant medication, compared with non-pharmacological treatments - improvement in cognitive deficits as well as reductions in symptom severity - may dissipate after a 2- to 3-year period, whether or not the medication component of treatment is continued or withdrawn (Molina et al, 
2009; Swanson et al, 2008a, b). In addition, the expected long-term relative benefits of childhood treatment with stimulants over non-pharmacological treatment on important non-symptom domains, such as substance use or delinquency that often emerge in ADHD individuals as they enter adolescence and adulthood, were not observed in the MTA follow-up (Molina et al, 2007, 2009).

Other long-term follow-up studies (Abikoff et al, 2004) suggest a different pattern of long-term residual benefits of medication. Powers et al (2008) recently reported on a longterm (about 9 year) follow-up of a cohort of 169 children with ADHD. Of these, 90 were assessed at follow-up, and 48 had a history of medication use for more than 1 year (average 5.33 years) and 42 had a history of no treatment or short-term treatment (less than 1 year). The self-selected subgroups differed on three measures of academic achievement and on grade point average, with the medication group outperforming the non-medication group, leading to the speculation that treatment with medication during childhood may improve long-term outcome in these cognitive domains. Biederman et al (2008) evaluated adolescent and adults from two large cohort studies of children with ADHD ( $n=140$ boys and $n=140$ girls, for a total $n=280$ ) and without ( $n=120$ boys and $n=122$ girls, for a total $n=242$ ). In the observational follow-up, in which about half of the sample was evaluated, some ADHD individuals were taking stimulant medication at the time of assessment $(n=26)$ and most were not $(n=94)$. The effects of medication were estimated by comparison of these two subgroups to each other and to controls $(n=133)$. The general pattern of performance indicated that, compared with the control group, the subgroup of ADHD not taking medication at follow-up had a more pervasive pattern of significant deficits than the subgroup of ADHD taking medication. A comparison of ADHD subgroups revealed that the subgroup on medication had better performance on sustained attention (measured by the CPT) and verbal learning (measured by the California Verbal Learning Test).

The developmental courses of behavioral manifestations of the disorder (symptoms) and neuropsychological performance (executive function deficits) have been evaluated in prospective studies of children with ADHD followed into adulthood, and some suggested attenuation in executive functioning deficits among patients with ADHD (Halperin and Schulz, 2006). However, the combined reports on neuropsychological testing of ADHD children present a highly inconsistent picture, with several studies failing to find differences on many measures of executive functions. Several meta-analyses designed to determine the degree to which executive function deficits can adequately account for the underlying cause of ADHD have failed to settle this question (see Homack and Riccio (2004), Huang-Pollock and Nigg (2003), and van Mourik et al (2005)). One interesting hypothesis is that the lingering ADHD-control discrepancy may be related to underlying heterogeneities in brain maturation trajectories. This hypothesis appears to be consistent with the observation that when a group of patients with childhood ADHD was subdivided based on their adolescent ADHD status, both persisters and remitters exhibited deficits in perceptual sensitivity and response variability, and fidgetiness (suggestive of an enduring subcortical impairment), whereas executive function deficits, relative to controls, were only seen in persisters (suggestive of a prefrontal cortex (PFC) maturation-related process of recovery) (Halperin et al, 2008).

A program of longitudinal brain imaging was established in the child psychiatry branch of the National Institute of Mental Health (NIMH), and the ADHD component of this program has provided evidence of a global reduction in brain volume, as well as multiple regional differences in brain anatomy, suggesting an approximately $10 \%$ reduction in prefrontal, basal ganglia, corpus callosum, and cerebellum (Castellanos et al, 2002a; Giedd et al, 2001; Shaw et al, 2006, 2007). Krain and Castellanos (2006) suggested that these brain deficits were non-progressive and associated with the disorder (presumably from the result of early genetic and/or environmental factors), rather than consequences of treatment of the disorder with stimulant drugs. Shaw et al (2006) and Shaw et al (2007) reported differences in brain development based on measures of cortical thickness, which was reduced in childhood in ADHD cases compared with controls. Both environmental (history of treatment with stimulant medication) and genetic (genotype of the dopamine (DA) receptor D4 gene) factors affected the developmental trajectory. Surprisingly, the group that had been treated consistently with stimulant medication appeared to show normalization in the trajectory of brain development (for a review see Shaw et al (2010)), but this group was self-selected and may differ on other factors related to outcome. The NIMH brain imaging program also provides the essential background of normal brain development in a large cohort of singletons (see Giedd et al (2001)) and twins (see Lenroot and Giedd (2008)), which have defined the pattern for typical brain development (ie, roughly linear increase in white matter volumes and inverted U-shaped trajectories for some gray matter structures), and suggest that some childhood onset disorders may be disorders of neuroplasticity (Rapoport and Gogtay, 2008). These reviews emphasize that the journey (trajectory) as well as the destination (end point) are important to consider in the evaluation of brain anatomy, and that the most important differences between normal and abnormal brain development are likely to be dependent on age and stage of development at the time of the evaluation.

In summary, we should note that the literature on longterm benefits of childhood treatment with stimulants is mixed. In some observational follow-up studies of children into adulthood, comparison of self-selected naturalistic subgroups suggest a long-term benefit of stimulant medication on cognition as well as behavior, but selection biases have not been adequately addressed to justify a firm conclusion about this hopeful hypothesis. The MTA study that used RCT methods documented temporary long-term effects that dissipated over time and with developmental 
course of $\mathrm{ADHD}$, so that there were no residual relative benefits of childhood treatment with stimulants. As a randomized trial of long-term treatment is impractical, controlled studies of neural adaptations produced by stimulants have been carried out in animals, and some long-term effects have been documented (Jezierski et al, 2007; Moll et al, 2001). Paired with appropriate cognitive tasks developed for the assessment of attention and impulsivity in animal studies (Winstanley et al, 2006), animal models could be developed to provide a rigorous evaluation of the long-term effects of stimulant drugs on brain development and cognition.

\section{THEORIES OF RESPONSE VARIABILITY AND DEFAULT MODE NETWORK}

\section{Evaluation of RT Distributions by Ex-Gaussian and Fast Fourier Analysis}

An influential review by Castellanos and Tannock (2002b) noted that '... response variability is one of the ubiquitous findings in ADHD research across a variety of speededreaction-time tasks, laboratories, and cultures' (p 624). In almost all studies that used RT as a measure of performance, ADHD individuals differ from controls in overall speed (slower average RT) and variability (increased within individual SD). In addition to these usual summary statistics, the shape of the distribution of RTs differs, because it is more skewed to the right in the ADHD individuals owing to an increased proportion of infrequent long RTs.

One hypothesis is that the skewness and infrequent long $\mathrm{RT}$ s are the result of periodic lapses in attention (Douglas et al, 1995). Leth-Steensen et al (2000) evaluated this theory with an innovative 'Ex-Gaussian' analysis of data from a spatial choice RT task. The usual RT analysis is based on a mathematical model of one underlying process, which generates a description of the empirical distribution of RTs provided by the mean and the first (SD) and second (skewness) moments about the mean. The 'Ex-Gaussian' model accounts for the empirical distribution of RTs based on an assumption of two underlying processes, with one generating a normal ('Gaussian') distribution of RTs described by the parameters $\mu$ (mu; mean) and $\sigma$ (sigma; standard deviation) and the other generating an exponential ('Ex') distribution of infrequent long response described by a parameter $\tau$ (tau; the tail of the empirical distribution). In this innovative study and analysis, the ADHD and control groups did not differ significantly in comparisons of the normal distribution parameter estimates of $\mu$ (575 vs $525 \mathrm{~ms}$ ) or $\sigma$ (86 vs $78 \mathrm{~ms}$ ), but they did differ for the exponential distribution parameter $\tau$ (354 vs $134 \mathrm{~ms}$ ). This suggested that the ADHD children had more lapses of attention marked by long RTs, but otherwise they responded about the same as the control children. The ADHD-control differences revealed by this 'Ex-Gaussian' analysis may depend on the task used. Hervey et al (2006) evaluated a group of stimulant-naïve ADHD children in the
MTA follow-up study $(n=65)$ and matched group classmates without ADHD $(n=65)$ using a Go-NoGo task (the Conners' CPT). On the basis of standard analyses, the ADHD group had a longer MRT and greater SD and skewness. However, an Ex-Gaussian analysis revealed that, compared with controls, the ADHD group had lower estimates of $\mu$ (ie, faster RTs) but a greater $\tau$ (ie, more long RTs). The former analysis would suggest inefficient responding (slow, inaccurate, and variable), whereas the latter analysis might suggest impulsive responding (fast and inaccurate) combined with interspersed long RTs owing to lapses of attention.

The Ex-Gaussian analysis has been applied to evaluate cognitive response to stimulant medication. Epstein et al (2006) evaluated the performance of 316 ADHD children in the MTA at the 2-year assessment, who were tested on the Conners CPT. At this assessment, 190 of the 316 (60\%) were taking stimulant medication and 126 (40\%) were not. A comparison of the self-selected subgroups evaluated by traditional analysis of RT (mean and SD) revealed that the subgroup on medication responded faster and was less variable than the subgroup off medication, but the Ex-Gaussian analysis revealed that this was owing to a reduction in $\tau$ (suggesting fewer long RTs in the inattentive state) and an increase in $\mu$ (slower RTs in the attentive state). Thus, the modeling of RT distributions by the ExGaussian approach leads to very different conclusions about the underlying effect of stimulant medication on cognitive processing.

Other methods have been developed to evaluate skewed distributions of RTs. Instead of randomly varying owing to either a normal or exponential process, Castellanos et al (2005) hypothesized that RTs varied systematically over time with a slow cycle of about 10-20s that reflected underlying variation at the synaptic level of the DA system (Walters et al, 2001). This systematically cyclical variation can be evaluated by using a fast Fourier transform (FFT) analyses of RTs, which also depend on the temporal characteristics of the task, such as the inter-trial interval (ITI) and the total time of a block of trials, that set the upper frequency (1/twice the ISI) and the lower frequency (1/half the duration of each block). Castellanos et al (2005) applied FFT analysis to evaluate RTs from the Eriksen-Flanker Task with an ITI $3 \mathrm{~s}$ and six trial blocks of $180 \mathrm{~s}$ each, which allowed for FFT decomposition into frequencies from 0.17 $(1 / 6)$ and $0.011 \mathrm{~Hz}(1 / 90)$. The usual summary statistics (mean and SD) suggested that the ADHD group had overall greater variability in responding, but the FFT analysis revealed that the magnitude of the systematic waxing and waning was greater for the ADHD than the control group (ie, oscillations centered at $0.05 \mathrm{~Hz}$ were present in both groups, but the power at this frequency was $50 \%$ greater for the ADHD than the control group). Following this innovation, others have used FTT analysis of RTs in studies of cognitive deficits of ADHD. For example, Di Martino et al (2008) used RTs from an Eriksen-Flanker task with a single 15 min block of trials, Vaurio et al (2009) used RTs from a 
Go-NoGO task, and Johnson et al (2008) used RTs from a sustained attention to response task (SART) that requires response to a fixed sequence of digits (1-9), except for the digit 3. In general, FFT analyses of these studies revealed that the ADHD group differed from the controls owing to an increase in power in the frequency band reflecting periodic cycles about every 10-20 s (as hypothesized by Castellanos) that increase variance of the RT distribution.

The FFT analysis has also been used to evaluate response to stimulant medication. Castellanos et al (2009) used FFT analysis to evaluate the effects of stimulant medication on performance on the Eriksen-Flanker task (see above), and showed that the group difference observed at baseline (greater amplitude of oscillations centered at $0.05 \mathrm{~Hz}$ in ADHD) was no longer significant with medication. Johnson et al (2008) used FFT analysis of RTs from the SART (see above) and showed that treatment with medication (and average titrated dose of $0.51 \mathrm{mg} / \mathrm{kg}$ ) over 6 weeks produced a decrease in the fast component of variability of RT.

In summary, we should emphasize that one of the most prominent cognitive deficits manifested in RT studies is increased variability, and that models of RT analysis have been applied to investigate underlying processes, such as randomly occurring lapses of attention (the Ex-Gaussian model) or systematic waxing and waning of attention (the FFT model) that affect RT. Task characteristics affect RT distributions and analyses, but this additional source of variation may be informative of the underlying cognitive processes. These models of analysis of RT distributions have been applied to evaluate effects of stimulant medication on cognitive processes such as attentional lapses ('mind wandering') or fundamental cyclical variation related to neurochemical processes and reflected in brain states, which has been addressed in the related concepts of 'resting state' or 'default mode' of brain function.

\section{History of the Concept of the Resting State and Default Mode Network}

Sonuga-Barke and Castellanos (2007) proposed that the skewed RT distributions of individuals with ADHD (see above) may be a consequence of interference by the default mode network (DMN) of the brain. Owing to the importance of DMN, as well as controversies about it, we will provide a brief review of this concept here.

Raichle (2010) provides a historical account of PET studies that led to the discovery of a 'constellation of brain regions now generally referred to as the DMN' ( $p$ 182). Breakthrough studies are reviewed that link the PET studies of the DMN to fMRI studies, which revealed 'spatial coherence in the spontaneous fluctuations (ie, noise) in the fMRI blood oxygen level-dependent (BOLD) signal'. As shown in Figure 1a, these brain regions-posterior cingulate cortex (PCC)/precuneus, medial prefrontal (MPF), and the lateral parietal cortex (LP) - have correlated increases in BOLD activity during the resting state when a task is not being performed shown in slow wave $(0.1 \mathrm{~Hz})$ cycles. Fox and Raichle (2007) provide a review of resting state fMRI studies, which consistently show within-subject positive correlations of the slow cyclical fluctuations of BOLD signals from the brain regions within the DMN (ie, PCC, MPF, and LP - see the red and yellow lines in Figure $1 \mathrm{~b}$ ).

Brain regions that are typically activated by task performance also show highly correlated cyclical fluctuations in BOLD signals (or functional connectivity), although this activity is out of phase with the waxing and waning of the BOLD signals from the DMN. Fox and Raichle (2007) used as an example a task-positive network related to focused attention and working memory that during the resting state is associated with opposing (anticorrelated) decreases in activity in the intraparietal sulcus (IPS), frontal eye field (FEF), and middle temporal (MT) brain regions (see the blue line in Figure 1a). The intrinsic brain activity in the task-positive network is out of phase with the intrinsic activity of the DMN, so the correlation of the spontaneous cyclical activity in the two networks over time is high-but negative or anticorrelated (see Figure 1b). Dosenbach et al (2007) described a dual-process taskpositive network consisting of a frontoparietal component that initiates and adjusts control and a cingulate-opercular component that maintains task orientation over time and '...might in part support a basic domain-independent and externally directed 'task mode' in opposition to the brain's
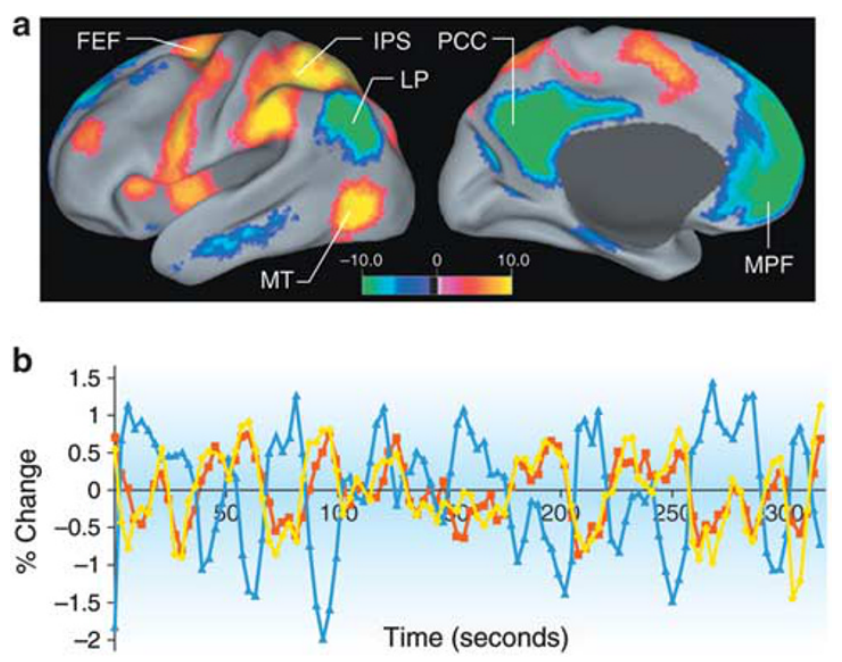

Figure 1. Anticorrelated brain activity in a component of the taskpositive network related to externally cued attention. (a) The intraparietal sulcus (IPS), frontal eye field (FEF) and middle temporal (MT) areas are the positive nodes (shown in warm colors) and are significantly correlated with seed regions involved in focused attention and working memory (task-positive seeds). The task-positive seeds are significantly anticorrelated with seed regions that are routinely de-activated during attention-demanding cognitive tasks (task-negative seeds shown in cool colors) and located in the posterior cingulate cortex (PCC)/precuneus, lateral parietal cortex (LP), and medial prefrontal cortex (MPF). (b) The bottom actograms represent the time course for a single run based on the seed region (PCC, in yellow), a region positively correlated with this seed region in the MPF (orange), and a region negatively correlated with the seed region in the IPS (blue). Modified with permission from Fox and Raichle (2007) and Fox et al (2005). 
default mode' ( $p$ 11076). On the basis of this, we will use the label 'task mode network' (TMN) for comparisons to the DMN. Of course, there are multiple TMNs dependent on the cognitive demands of tasks, and each may be related (or anticorrelated) with the same or different DMNs. For example, Fox et al (2006) proposed dorsal-ventral distinctions between a dorsal TMN and ventral DMN. The proposed TMN included the dorsal-lateral and dorsalventral prefrontal brain regions that are activated by a variety of cognitive tasks. The proposed ventral network (equivalent to the DMN) included the MPF, posterior cingulate, and lateral parietal brain regions, which are assumed to be activated when not performing a task (in the 'resting state'). Fransson (2006) presented a similar conceptual framework of cognitive control and proposed the brain switches ('toggles') between the DMN and TMN. De Luca et al (2006) identified five resting state networks (RSNs), and one of these (RSN-2) was equivalent to the DMN described by Raichle (2010) and Fox et al (2006a) and depicted in Figure 1.

There is evidence from several studies that spontaneous BOLD fluctuations during task performance are related to trial-to-trial variability in task-related evoked signals (Fox et al, 2006b; Fransson, 2006). This suggested the exciting possibility of linking temporal variation in brain states to variability in behavior or performance on a task, such as variability in force applied in a key press response task (Fox and Raichle, 2007) or variability in RT (Weissman et al, 2006). For the purposes of the current review, the most relevant demonstration of the cyclical anticorrelated fluctuations of BOLD activity in the DMN and TMN is provided by Kelly et al (2008). The brain regions emphasized by Kelly et al (2008) and the pattern of negative correlation between BOLD activity in the DMN and the TMN for the Eriksen-Flanker Task are shown in Figure 2a.
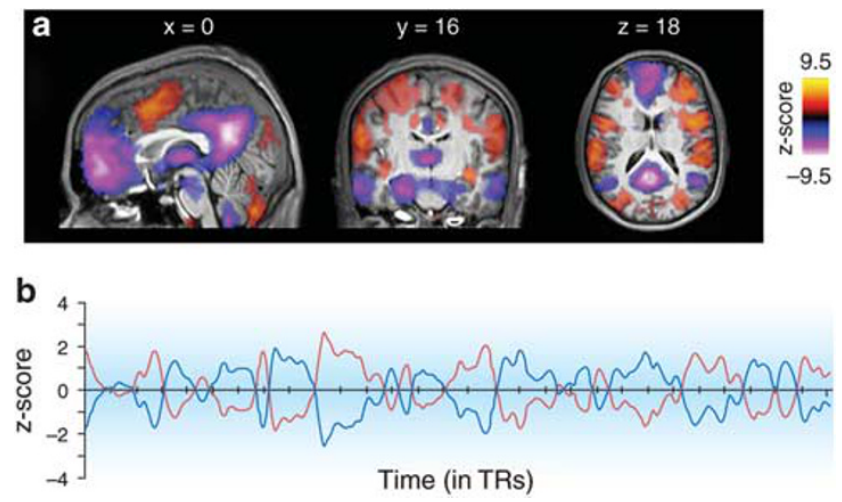

Figure 2. The pattern of negative correlation between blood oxygen level-dependent (BOLD) activity in the default mode network (DMN) (equivalent to RSN-2) and the task-positive network for a component of the attentional network task (ANT) task. (a) Z-score threshold maps of the spontaneously active (task-independent) DMN (purple/pink) and its negatively correlated task mode network (TMN) (red/orange). (b) An individual example of a nearly perfect negative correlation $(r=-0.97)$ between DMN and TMN antiphase time series. Reprinted with permission from Kelly et al (2008).
High negative correlations (see Figure $2 \mathrm{~b}$ for an individual example) were observed for all of the 26 subjects evaluated, with a range from -0.80 to -0.97 , and this inter-subject variation in the degree of anticorrelation was related to inter-subject variation in performance measures of the task (RT variance): the subjects with the higher anticorrelations had lower RT variances in performance of the task.

It is important to mention that raw fMRI data from the resting state must be edited to remove contaminating motion artifacts and various other sources of physiological noise. Some experts have suggested that these techniques might introduce spurious negative correlations upon regressing out whole brain signals (Murphy et al, 2009; Van Dijk et al, 2009). Beyond the technical questions, it is necessary to contend with the claim that '...the observation of a difference between a subtraction and a reverse subtraction is not evidence for the importance of the baseline' (Morcom and Fletcher, 2007). Clearly, the use of global regression techniques warrants extra caution when interpreting the directionality, or even the meaning of resting state relationships. On the other hand, it has been pointed out that several of the characteristics of anticorrelated networks (eg, spatial distribution, cross-subject consistency, presence with modified whole brain masks, and existence before global regression) cannot be attributed readily to global signal removal, which supports the notion that these signals represent a true underlying biological phenomenon instead of a statistical artifact (Fox et al, 2009).

Despite these caveats and concerns, in summary we should emphasize that the discovery of the intrinsic organization of brain activity and the development of fMRI methods to characterize networks and functional connectivity offer the exciting possibility of an important new way to map brain organization and to investigate disorders (such as ADHD). It is important to recognize that this can be accomplished without the use of a task (ie, in the socalled resting state) as well as with a variety of tasks. This DMN approach is consistent with a hierarchical organization of brain networks (Raichle, 2010): the DMN may be a 'hub' at the top of a hierarchal organization, which operates '...to organize information for interpreting, responding to, and even predicting environmental events'. In this organization it is assumed that this '....activity is modulated by phase resetting to match incoming information, to increase activity in brain areas associated with goal-directed behavior, and to enhance performance on cognitive tasks.'

\section{Theoretical Approach and Empirical Tests in the ADHD Area}

Sonuga-Barke and Castellanos (2007) used the concept of anticorrelated networks (which we have outlined in Figures 1 and 2) to propose an alternative to the traditional topdown model of ADHD based on executive function deficits (which we reviewed in 'Background studies on the effects of stimulants in cognition in ADHD' above). During the 
resting state, this theory proposes a recurrent toggling or switching (Fransson, 2005) between a state of self-reflection (an 'Introspective' state) and a state of attentive readiness (an 'Extrospective' state). Sonuga-Barke and Castellanos (2007)) proposed the default-mode interference hypothesis to account for the systematic increased intraindividual variance in RT tasks (which we have reviewed in 'History of the concept of the resting state and default mode network' above). They considered the task-positive and task-negative networks together as a default mode state in which spontaneous fluctuations are synchronized in the opposing (anticorrelated) relationship described in Figure 1. This produces a corresponding cyclical pattern of Introspective and Extrospective states. When a task is initiated, they propose the magnitude of the fluctuations in Introspective and Extrospective states is decreased but, over time, this magnitude increases, crossing a threshold at some point that they label the 'default mode interference threshold'. When this occurs, they propose a lapse of attention results in a long RT. In individuals with ADHD, the recovery and 'default mode interference' is faster and the lapses of attention are more frequent than in non-ADHD individual when performing a task (such as the Go-NoGo, STOP, or ANT), resulting in an increase in the intraindividual variance of RTs that creates a skewed distribution.

A few recent $\mathrm{FMRI}$ studies of ADHD and the intrinsic activity in the resting state provide data that can be used to evaluate the default mode interference hypothesis. Tian et al (2006) evaluated resting-state functional connectivity in 12 adolescents (11-15 years of age) with ADHD and 12 controls based on correlations with the dorsal anterior cingulate gyrus shown to function abnormally in task-based fMRI studies. The ADHD group had greater resting state connectivity than the controls in multiple brain regions (bilaterally in the dorsal anterior cingulate, pons, insula, cerebellum, and thalamus), which was interpreted as abnormalities in areas involved in autonomic control. Zang et al (2007) evaluated 13 adolescents with ADHD and an age-matched control group (average age about 13 years), and reported increased functional connectivity for some brain regions (right anterior cingulate gyrus, left cerebrum/fusiform, right inferior temporal gyrus, left sensorimotor cortext, and bilateral brain stem regions in the midbrain and pons) but decreased on others (right inferior cortex, bilateral cerebrum, and cerebellar vermis). An observed increase in right anterior cingulate gyrus activity in the resting state was particularly interesting because it suggested the opposite of the expected ADHDrelated deficit (decreased activity) when performing a task. Similarly, Yang et al (2010)) also evaluated children in a resting state fMRI study and reported that, compared to controls, the ADHD group had specific brain regions displaying either higher and lower functional connectivity. Even though these group differences may be compounded by large differences in IQ, the nature of the regions involved could be tentatively interpreted as a reflection of abnormalities in intrinsic brain organization in the frontal cortex in
ADHD children in the resting state. Castellanos et al (2008) found that a group of adults with ADHD had reduced negative correlation with a region in the DMN (precuneus/ posterior cingulate gyrus). This decreased functional connectivity during the resting (which partially contradicts Tian et al (2006), who reported increased functional connectivity between the dorsal anterior cingulate and widespread regions involved in autonomic control) was interpreted as an abnormality in the interactions between the TMN and DMN. This may underlie performance deficits in the task state - attentional lapses and the periodic long RTs that characterize the performance of individuals with ADHD when they perform a task. Uddin et al (2008) also study the DMN in the same sample of adults with ADHD and found that, compared to controls, network homogeneity was lower for one region (the precuneus). This was interpreted as abnormal function of a highly integrative structure with connections to many cortical (eg, anterior cingulate gyrus) and subcortical (eg, striatum) brain regions implicated in ADHD.

Fassbender and Schweitzer (2006) evaluated functional connectivity during the performance of tasks (a visual serial visual search task, an addition task, and a matching to sample task). They investigated the persistence of anticorrelated BOLD activity in task-negative networks (ie, the DMN) during task performance and the ability to deactivate the task-negative DMN during task performance that is reflected in TMNs. They compared the RT performance of 12 ADHD and 12 control children, which by Ex-Gaussian analysis showed the skewness presumed to occur owing to periodic lapses of attention. The groups differed in the degree of deactivation of the DMN: during performance of the most demanding (visual search), the ADHD group showed less deactivation of the medial PFC than the control group. The ADHD group also showed greater intraindividual variability, which was negatively correlated with activity in the PCC. This was interpreted as a relative weakness in the ADHD individuals to suppress activity in the DMN during performance of a working memory task, and it provided support for the hypothesis of Sonuga-Barke and Castellanos (2007) of default mode interference as a cause of periodic attentional lapses and long RTs.

Wolf et al (2009) evaluated 12 adults with ADHD and 12 age-matched controls during performance of a three-phase (encoding, delay, and probe) working memory task. The groups did not differ in performance of this task, but they did differ in the patterns of brain activation elicited by the task (ie, in the task-positive networks for working memory) and in the functional connectivity estimates during the delay phase of the task. The ADHD group had less functional connectivity for some brain regions (ventrallateral PFC, anterior cingulate gyrus, superior parietal, and cerebellum) and greater functional connectivity for other brain regions (right PFC and left dorsal anterior cingulate gyrus, and cuneus). This was interpreted as consistent with the hypothesis of ADHD-related dysfunction of the prefrontal-parietal, anterior cingulate, and cerebellar brain 
regions proposed based on anatomical and functional imaging studies that we outlined in the Introduction (Bush, 2010; Castellanos and Tannock, 2002b).

Peterson et al (2009) used the Stroop task in an fMRI study of children without ADHD $(n=20)$ and children with ADHD $(n=16)$ evaluated on and off of their established clinical doses of stimulant medication. They found significant differences in brain activation for the ADHD group off medication compared with the control group, suggesting that the ADHD group was not able to suppress activity in the DMN to the same degree as the control group, and in the ADHD group medication appeared to improve the suppression of DMN activity in the ventral anterior cingulate gyrus.

In summary, we should relate the literature on variability of RTs and the literature on functional connectivity. The literature based on multiple RT paradigms suggests that ADHD individuals have an increased proportion of infrequent long reactions that skew the distribution of RTs and may reflect lapses of attention. The literature on brain states suggests that spontaneous cyclical activity is correlated across brain regions related to cognition, and is organized in opposing TMN and DMN (ie, task-positive and task-negative networks - see Figures 1 and 2). The BOLD activity from fMRI signals may be related to variation in RTs. In ADHD, abnormalities in functional connectivity have been documented, but both increases and decreases relative to controls have been observed. Increased activity in the DMN was present in some studies, which is consistent with the hypothesis that ADHD individuals may have increased intrusions during task performance that are manifested as lapses of attention and variable patterns of response that reflect in part improper deactivation of the DMN. Stimulant medication may facilitate the deactivation of the DMN and alleviate the ADHD symptoms of inattention and their variable manifestation over time.

\section{INTEGRATION OF ATTENTION AND MOTIVATION}

\section{Attention}

In the previous sections, we discussed this evidence for attentional deficits in ADHD from the perspective of the cognitive neurosciences. We have focused on the impaired ability to suppress ongoing actions or pre-potent responses that is one of the hallmarks of the theory of a core inhibition deficit of ADHD (Barkley, 1997) and is one of the most disruptive aspects of ADHD (Kenemans et al, 2005). In addition, we have also explored a variety of deficits manifested in three cognitive components of attention (alerting, orienting, and executive control) linked to specific neural loci (right frontal, parietal, and anterior cingulate gyrus) and neurotransmitters (norepinephrine, acetylcholine, and DA (see (Posner and Rothbart (2009)). Another model of attention proposes two networks; a dorsal network involved in top-down orienting of attention and a ventral attention network involved in reorienting attention in response to salient sensory stimuli (Corbetta and Shulman, 2002; Fox et al, 2006a). As discussed above, the ventral attention network orients attention to internal or Introspective processes and is part of the DMN that is activated in the so-called resting state (see discussions in 'Background studies on the effects of stimulants in cognition in ADHD' and 'Theories of response variability and default mode network' and Figure 1). The DMN is deactivated during cognitive operations but not completely suppressed, as the dorsal TMN is activated (see Figure 2), resulting in anticorrelation of BOLD activity even during the task state (Fox et al, 2006a). To evaluate cognitive deficits in $\mathrm{ADHD}$, both Introspective and Extrospective processes appear to be important, and neuropsychological and brain imaging methods are being developed to characterize ADHD-related cognitive deficits and effects of stimulant medication that may correct them (see Sonuga-Barke and Castellanos (2007)).

\section{Motivation}

Motivation and reward may represent another 'core' deficit of ADHD (Luman et al, 2005). The notion that ADHD involves an underlying dysfunction in reward and motivation was proposed over two decades ago (Haenlein and Caul, 1987), revived a decade ago (Iaboni et al, 1997), and emphasized in some current theories of ADHD (see below). Several new theories of motivational deficits have been proposed over the past decade. Johansen et al (2002) proposed a theory based on the premise that ADHD children have a steeper than normal delay-of-reinforcement gradient, and Sonuga-Barke (2002) proposed a theory based on the premise that some ADHD children have an aversion to delay of reinforcement.

Luman et al (2005) evaluated the frequency and magnitude of reinforcement on a learning task by contrasting performance of ADHD children $(n=23)$ and controls $(n=30)$, as well as a clinical control group of children with autistic spectrum disorder $(n=21)$. The ADHD group was unaffected by the manipulation of reinforcement by frequency or magnitude of reinforcers. Bitsakou et al (2009) evaluated delay aversion in children with ADHD $(n=70)$, a control group of unaffected siblings $(n=65)$, and a normal control group $(n=50)$. Multiple tasks were administered and an overall index of delay aversion was used to contrast the groups. The contrasts revealed a significant difference between the ADHD and normal control group (es $=0.9$ ), and even though the difference compared to the familial control group was not significant, the average for the sibling control fell midway between the average for the ADHD and the normal control groups. Shanahan et al (2008) evaluated children with ADHD $(n=25)$ and normal controls $(n=30)$ using the STOP task with and without motivation. This study documented significant main effects of motivation on overall RT, variability of RT, and SSRT, but the critical group by motivation interaction was not significant. Shiels et al (2008) tested children with ADHD $(n=21)$ in 
a crossover design to evaluate the effects of a behavior modification technique (point system with back-up reinforcers) on a spatial span task that required storage (forward span) and manipulation (backward span). Motivational incentives enhanced performance on the backward span, but not the forward span task. In an epidemiological study of 1156 children, Kuntsi et al (2009) used a Go-NoGo task to evaluate $\mathrm{RT}$ and $\mathrm{RT}$ variability in the $5 \%$ of children with the highest ratings of ADHD $(n=58)$ compared with the remainder of the population sample $(n=1098)$. In the baseline task, with a slow event rate ( $8 \mathrm{~s})$, the ADHD group had longer average $\mathrm{RT}$ and higher intraindividual variability (average mean $\mathrm{RT}=1018 v s 943 \mathrm{~ms}$; average $\mathrm{SD}=495 v s$ $404 \mathrm{~ms}$ ). To improve performance on the task, a fast event rate $(1 \mathrm{~s})$ was used and incentives were provided by a point system (1 point earned for correct responses, 1 point lost for omission errors, and 5 points lost for commission errors, with a prize as back-up reinforcement). Compared with the performance on the task with a slow event rate and no incentive, the combination of a fast event rate and the incentive condition normalized speed of response (mean $\mathrm{RT}=653$ vs $648 \mathrm{~ms}$ ) and intraindividual variability ( $\mathrm{SD}=217$ vs $203 \mathrm{~ms}$ ).

A few recent studies evaluated the effects of medication and motivation (reinforcement) on brain function. For example, Rubia et al (2009) evaluated children with ADHD $(n=13)$ and normal controls $(n=13)$ on CPT task performed in an fMRI study of 'cool' attentional and 'hot' motivational brain networks. The CPT had 48 occurrences of two targets (24 each of the letters $\mathrm{X}$ and $\mathrm{O}$ ) embedded in 416 trials, and correct responses to one of the targets was rewarded with money. Off medication, the ADHD group had significant performance deficits on CPT, and reduced activation of a brain network that connected frontal, striatal, parietal, and cerebellar brain regions and increased activation of orbitofrontal and superior temporal brain regions. Medication normalized the attentional network (upregulating activity) and in the motivational network (downregulating reward-induced overactivity). A recent neuroanatomical study (Carmona et al, 2009) provided clear evidence of alterations in the ventral striatum of children with ADHD. This finding is consistent with recent fMRI data showing ventrostriatal hyporesponsiveness during immediate and delayed reward processing in adults with ADHD (Plichta et al, 2009; Strohle et al, 2008). Tripp and Wickens (2008) proposed the DA transfer deficit theory and suggested that children with ADHD have diminished cellular responses of DA cells to cues that precede reinforcement.

In summary, we should emphasize that multiple theoretical approaches have emerged in the literature that suggest that ADHD children manifest impulsive behavior that is characterized by preference for small immediate reinforcers over large delays reinforcers (see reviews by Johansen et al (2009) and Luman et al (2005)). Recently, the emerging critical issues in this area of motivation or reinforcement deficits in ADHD were addressed at the European Network of Hyperkinetic Disorders (EUNETHYDIS) 2009 Conference (Sergeant $e t a l, 2009$ ) and in an international workshop at Okinawa Institute of Science and Technology (Tripp and Wickens, 2009). Another comprehensive review would be premature, but studies in this area are increasing, and this represents a new direction for the evaluation of cognitive deficits in ADHD and the possible correction of these deficits by stimulant medication.

\section{Neuronal Targets of Stimulant Medications}

Several brain regions and circuits are involved in the orchestration of specific cognitive processes that impact attention; these circuits are modulated by the catecholaminergic system and thus are affected and modulated by stimulant medications (Wilens, 2008). Most of our knowledge about the mediation of the therapeutic effects of stimulants derives from studies focused on the PFC and striatum, but there is increasing evidence of the relevance of catecholaminergic modulation in limbic regions such as ventral striatum (perhaps also amygdala and hippocampus) in mediating the motivation enhancing effects of stimulant drugs (Lehmann et al, 2003; Wilens, 2008).

As reviews of brain imaging studies document (see Paloyelis et al, (2007) for a systematic review), many studies that compare ADHD and control groups have documented a long list of possible anatomical (Krain and Castellanos, 2006) and functional (Dickstein et al, 2006) abnormalities associated with ADHD. As reviewed and discussed in 'Background studies on the effects of stimulants in cognition in ADHD', the most consistent patterns include abnormalities revealed by anatomical MRI (ie, reduced global size as well as brain regions in frontal lobes, basal ganglia, and cerebellum (Krain and Castellanos, 2006), and by functional MRI (ie, frontal hypoactivity affecting anterior cingulate, dorsolateral prefrontal and inferior PFCs, portion of the parietal cortex, as well as basal ganglia and thalamus; see Dickstein et al (2006)). Moreover, recent studies have shown that disruptions in ADHD affect not only the activity in these brain regions but also the way in which these regions connect with one another to form specific networks. The fMRI methods are highlighted in Figures 1 and 2 and discussed in detail in 'Theories of response variability and default mode network'. In the next section, we will discuss PET methods that address the evaluation of neural mechanisms related to the cognitive deficits in ADHD and their possible correction by treatment with stimulant medication.

\section{PET IMAGING AND COGNITIVE PROCESSES IMPLICATED IN ADHD AND EFFECTS OF STIMULANT DRUGS}

\section{Neural Deficits Related to ADHD}

Patients with ADHD present with specific cognitive deficits, which may be the consequence of impaired attention or 
motivation or both. In previous sections, we based our interpretations on the literatures on neuropsychological performance (see 'Background studies on the effects of stimulants in cognition in ADHD') and fMRI imaging of functional activation and functional connectivity in tasknegative and task-positive networks of the brain (ie, the DMN and TMN - see 'Theories of response variability and default mode network'). On the basis of the PET imaging approach, the literature and concepts are somewhat different. Here, we discuss the neurobiological substrates underlying the cognitive processes evaluated by PET imaging, which have contributed considerably to our knowledge on how stimulants may modulate them.

PET studies have investigated the mechanisms of DA transmission and their relationships to the hyperactiveimpulsive and inattentive symptoms of children with ADHD. The DA hypothesis of ADHD was proposed decades ago (Levy, 1991; Wender, 1971), based partially on the hypothesis that stimulants acted as DA agonist drugs and that the increase in synaptic DA was a critical factor in the response to stimulant medication. PET study confirmed this hypothesis, first with intravenous doses of MP (Volkow et al, 1995, 1999a) and then with oral clinical doses of MP (Swanson et al, 1999; Volkow et al, 1999b, 2002).

Surprisingly, it is still unclear and debated whether DA activity is enhanced or depressed in individuals with ADHD. For the past decade, a generally accepted underpinning of biological theories of ADHD has been that this disorder is associated with abnormally high levels of DAT density, which were suggested by studies using single photon emission tomography to evaluate small samples of ADHD adults (Dougherty et al, 1999; Krause, 2008). These studies were based on the hypothesis that high DAT density would accelerate reuptake of synaptic DA and create a DA deficit. Some recent PET studies seem to corroborate these early findings: Larisch et al (2006) reported a modest increase in striatal DAT density in adults with ADHD, and a similar study (la Fougere et al, 2006) reported heterogeneous distribution, including high DAT densities. In one of the largest studies so far, Spencer et al (2007) evaluated unmedicated ADHD adults and reported striatal binding potential was at $2 \mathrm{SD}$ above the mean for the control group.

Some studies suggest the reverse. For example, in a PET study Jucaite et al (2005) observed reduced DAT binding in the midbrain of adolescents with ADHD. In a series of PET studies using the radioligands $\mathrm{C}^{11}$ raclopride and $\mathrm{C}^{11} \mathrm{co}-$ caine, Volkow et al (2007) assessed the density of DAT and DA D2/D3 receptors in the striatum of stimulant-naïve adults with ADHD. Individual differences in MP-induced increases in synaptic DA were not related to individual differences in DAT density (Volkow et al, 2002), suggesting that DA release rather than the DA reuptake may be a primary factor contributing to a DA deficit in the ADHD brain and the manifestations of deficits in attention. Volkow et al (2009b) found lower DAT and D2/D3 receptor density in the nucleus accumbens as well as the caudate nucleus. In a prospective long-term treatment study of MP (Wang et al,
2009), DAT density was reassessed in a subset of these ADHD patients after 1 year, with a 24-h washout to avoid the confusion of DAT density with DAT occupancy by MP. The formerly decreased DAT density was now increased in the same individuals, suggesting that neuroplasticity may operate in a homeostatic way to maintain DA levels in a narrow range: in response to high synaptic DA that results from blockade of DAT by clinical oral doses of MP (Volkow et al, 1999b, 2002), DAT density may increase and be a consequence of the DA agonist effect of MP. This may contribute to acute tolerance (Swanson et al, 1999; Volkow et al, 1998, 2001, 2002) and possibly long-term tolerance (Coghill et al, 2007; Vitiello et al, 2001) to clinical doses of stimulant medications.

In summary, the literature on PET imaging supports the DA hypothesis of ADHD, but the specific details are not yet clear. Some studies support the DAT excess hypothesis, which results in a DA deficit owing to increased reuptake of synaptic DA, whereas others support the hypothesis of DAT plasticity that resets density based on levels of synaptic DA, which may be low in stimulant-naïve individuals but high in treated individuals with clinical doses of MP as a result of adaptation to treatment rather than the presence of the disorder.

\section{PET Studies of Attention and Motivation}

On the basis of PET imaging studies, it has been hypothesized that stimulant medications may act by facilitating the engagement of a dorsal task-positive attention network and the deactivation of the ventral resting state network or DMN (Volkow et al, 2008). This may reflect in part improved filtering out of task-irrelevant stimuli by way of stimulant-mediated DA and NE release in the PFC and anterior cingulate gyrus (Arnsten, 2006). Other PET imaging studies of effects of stimulant medications on the brain have shown increased regional blood flow in the anterior cingulate gyrus (Udo de Haes et al, 2007). Also, PET studies have suggested that non-responsiveness to stimulant medications could be partially explained by different patterns of cerebral blood flow in anterior cingulate gyrus (Cho et al, 2007).

As a result of their ability to increase DA, stimulants appear to enhance the motivational saliency of cognitive tasks. On the basis of findings from a PET study that showed a significant association between MP-induced DA increases in the striatum and the perception as to how interesting the task was, Volkow et al (2004) postulated that stimulant's therapeutic effects may be mediated in part by their ability to enhance motivation. Increased interest may explain why stimulants improve performance of a boring task in normal healthy individuals as well as in ADHD individuals (Robbins and Sahakian, 1979; Sahakian and Robbins, 1977) and why unmedicated children with ADHD are able to perform properly when the task is salient to them (Groom et al, 2010). 
In summary, we should note that recent findings from PET brain imaging studies have documented that the DA deficits in ADHD were most prominent in the ventral striatum (a crucial brain region for modulating reward and motivation) and in the midbrain (where most DA neurons are located), which highlights the relevance of the reward/ motivational circuit in this disorder (Volkow et al, 2009b).

\section{PET Imaging Studies of ADHD Adults and Effect of Stimulant Drugs}

The effects of MP at its site of action in the brain have been studied extensively with PET. Imaging studies have corroborated the ability of oral MP to induce significant levels of DAT blockade in the striatum, with an estimated ED50 (dose required to block $50 \%$ of the DAT) of $0.25 \mathrm{mg} / \mathrm{kg}$ (Volkow et al, 1999a). Imaging studies have also shown that MP and AMP, at clinically relevant doses, can significantly increase extracellular DA (Villemagne et al, 1999; Volkow et al, 1999b, 2009a). Imaging studies in non-human primates have also shown significant blockade of the NE transporter at doses that are pharmacologically equivalent to those used in humans, which is interesting in light of the data indicating that enhanced extracellular catecholamine levels in cortical regions, secondary to $\mathrm{NE}$ reuptake inhibition, improves multiple aspects of inhibitory control over responding in rats and monkeys (Seu et al, 2009). Synaptic levels of DA and NE, under physiological conditions, act primarily as neuromodulators changing the efficacy of other transmitter signals (Keeler et al, 1989; Kiyatkin, 2002) as a function of ongoing neuronal activity (Seamans and Yang, 2004). For example, in the striatum, applications of DA decrease the activity of spontaneously active neurons to a greater extent than that of glutamatestimulated neurons (Kiyatkin and Rebec, 1996). These increases in glutamate-induced excitation relative to baseline are assumed to improve signal-to-noise neuronal activation (Rolls et al, 1984). Norepinephrine can also facilitate excitatory transmission by depressing the level of basal activity (Woodward et al, 1979). Thus, by virtue of their DA and NE amplifying capacity (Kuczenski and Segal, 1997; Solanto, 1998), stimulants may enhance task-specific signaling in target neurons within specific circuits.

By enhancing signal-to-noise ratio of neuronal activity, stimulant medications may render the brain more efficient.
And there is evidence from brain imaging studies suggesting that this may indeed be the case. For example, using PET and FDG, Volkow et al (2008) compared brain glucose metabolism in controls when performing a cognitive task (numerical calculations) with or without MP. As shown in Figure 3, compared with placebo, MP significantly reduced the amount of glucose utilized by the brain when performing the task (the increase was reduced by about $50 \%$ ). This reflected both a decrease in the magnitude of task-dependent regional activation and greater deactivation of regions from the DMN that has been described in Raichle (2010) and implicated in ADHD (Sonuga-Barke and Castellanos, 2007). This suggests that, compared with placebo, MP reduced (focused) the use of attentional resources in the human brain that are necessary to achieve similar levels of performance on a task. Moreover, subsequent PET studies that measured availability of DAT and functional activation/deactivation (measured with fMRI) during visual attention revealed a positive correlation between DAT and activity in the DMN, such that the higher the DAT, the less the deactivation of the DMN. As stimulants block DAT, this was interpreted to indicate that stimulant therapeutic actions reflect in part their ability to facilitate and sustain the deactivation of the DMN during task performance (Tomasi et al, 2009).

These findings are also consistent with those reported by previous PET imaging studies showing reductions with MP in the CBF increases in the dorsolateral PFC and posterior parietal cortex when controls performed a working memory task (Mehta et al, 2000). Similarly, imaging studies reported that MP reduced the CBF increases in the PFC when adults with ADHD performed a task of executive function (Schweitzer et al, 2004). Moreover, as recent studies provide evidence of deficits in the DMN in ADHD (Uddin et al, 2008), this may be a target for the therapeutic effects of stimulants.

Focusing of regional activation during task performance by stimulant medications may be beneficial when neuronal resources are diverted (ie, by 'mind-wandering') by the situation or task (Dodds et al, 2008) or by personality (Clatworthy et al, 2009), as well as by the presence of the disorder (ADHD). This may lead to an inverted U-shaped relationship between the effect of DA and performance, based on the premise that deficits as well as excesses of DA (and of NE) can impair cognition (see Figure 4). Thus, the
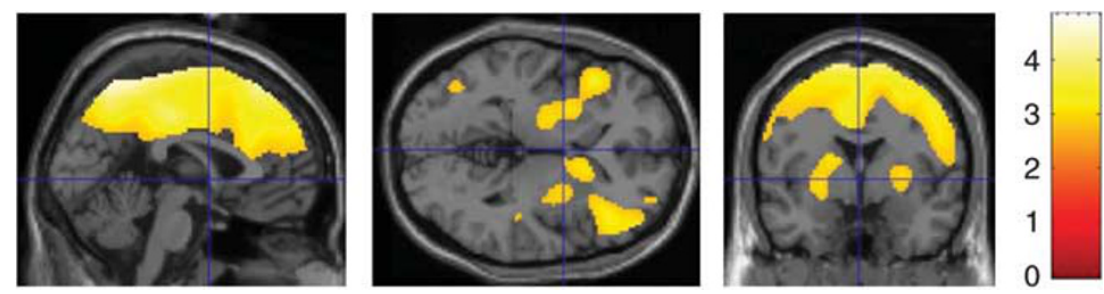

Figure 3. Differences in task activation between placebo (PL) and methylphenidate (MP). Statistical Parametric Mapping (SPM) results showing the areas (in yellow) that had greater increases in metabolism when the cognitive task was given with placebo vs when it was given with MP. Comparisons correspond to paired $t$-tests ( $p<0.005$ uncorrected $>100$ pixels). None of the brain regions had higher metabolism for the cognitive task when given with MP than with placebo. Reprinted with permission from Volkow et al (2008). 


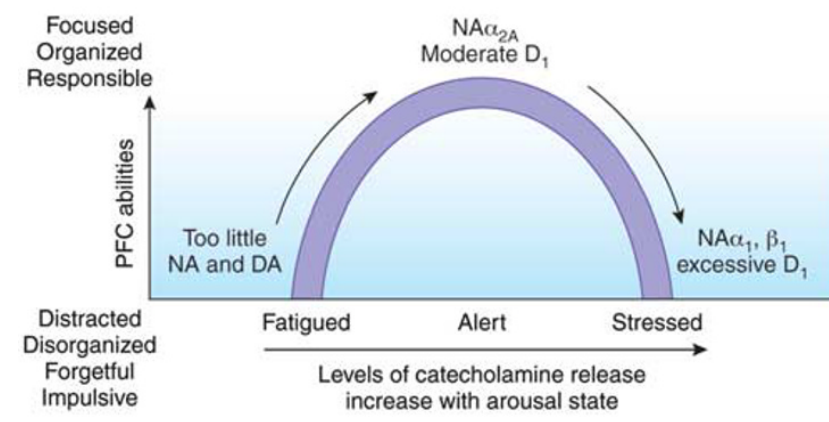

Figure 4. U-shaped curve relating activity on the prefrontal cortex (PFC) to levels of catecholamine release. As noted by Arnsten (2009), the PFC is very sensitive to catecholamine levels, with which it is suffused as a function of the arousal state. As proposed, PFC function is impaired whenever too little or too much catecholamine is released. In this schematic representation, moderate levels of noradrenaline (NA) and dopamine (DA) improve PFC function by optimally engaging their cognate receptors. Reprinted with permission from Arnsten (2009).

effects of stimulant drugs could be detrimental when brain activity is already optimally focused. This would also explain why stimulants may have beneficial effects in some individuals and not others, and beneficial effects in certain contexts and detrimental effects in others. Indeed, the inverted U-shaped relationship between cognitive performance and dopaminergic and noradrenergic signaling in frontostriatal circuits has been proposed to account for the intra- and interindividual differences in the effect of stimulant medications in ADHD patients. On the basis of animal models (Berridge et al, 2006; Robbins and Arnsten, 2009), the optimum alert state (see Figure 4) appears to be related to more moderate levels of activation of $\mathrm{NE} \alpha-2 \mathrm{~A}$ and DA D1 receptors in the PFC, but that stress-related increases of release of $\mathrm{NE}$ and $\mathrm{DA}$ and results in the activation of $\mathrm{NE} \alpha-1$ and $\beta-1$ receptor and excessive activation of D1 receptors (Arnsten, 2009).

Support for this hypothesis is provided by studies in laboratory animals, which have shown consistently that responses to stimulant drugs are determined in part by the state of the DA system (Deminiere et al, 1989; Hooks et al, 1991). Similarly, in laboratory animals, MP-induced increases in extracellular DA in the PFC are dependent on the conditions of its administration (Marsteller et al, 2002).

To the extent that the DA system (and possibly the noradrenergic system as well) is involved in ADHD, this could underlie the differential response to stimulant medications between controls and patients with ADHD. For example, in fMRI studies of children with ADHD (Vaidya et al, 1998) and of adults with ADHD (Epstein et al, 2007), MP's effects on striatal activation during performance of a task may recruit inhibitory control circuits that differ from those seen in healthy children. In a relevant fMRI study in non-ADHD adults, Dodds et al (2008) showed that the effects of MP on the brain depended on the task being performed, with attenuation of BOLD activity in the ventral striatum during phase of a reversal learning task (after negative feedback) and modulation of the BOLD signal in the PCF in the face of distractions when response set was maintained. The context dependency of stimulant effects has also been shown in humans. For example, a PET study showed MP-induced increases in extracellular DA were greater when healthy subjects were exposed to a salient stimulus (visual display of food in food-deprived individuals or with exposure to remunerated stimuli) than when given with a neutral stimulus (Volkow et al, 2002). Also, in a PET study Clatworthy et al (2009) documented variability in MP effects on subregions of the striatum and changes in D2/D3 receptor for different tasks requiring reversal learning and spatial working memory.

\section{FUTURE DIRECTIONS AND CONCLUSIONS}

As knowledge with regard to human brain function increases, we should gain a better understanding of the various neuronal components implicated in functional deficits in ADHD (attention, inhibition and motivation) and how stimulant medications affect them. This, in turn will permit a much better characterization of the specific deficit(s) in any given ADHD patient. The advances in the definitions of cognitive deficits in ADHD and cognitive effects of stimulant medication (see 'Background studies on the effects of stimulants in cognition in ADHD') set the stage for a future when treatment might be personalized to target the specific deficits of a patient.

The literature suggests a few important directions to pursue. For example, it is intriguing that stimulant medications appear to have different effects depending on the context of administration and the type of task required, as it has been shown for classical neuropsychological tasks that require reversal learning and spatial working memory (Clatworthy et al, 2009) or responses within a task such as reversal learning (Dodds et al, 2008). It is also likely that fast shifting tasks with rewards (eg, a video game) (Koepp et al, 1998) may induce, by themselves, larger DA increases than slower tasks (memory or composition) that require maintenance of attention, so that these two types of tasks are likely to be differentially affected by stimulants. On the basis of the likelihood of a ceiling effect, it is reasonable to propose that stimulant medication would lead to a greater improvement in the performance of the latter compared with the former tasks. Also, the literature of RT distributions clearly shows context effects that alter the peak frequency of slow wave variations over time. Stimulants may also shift the engagement of attentional resources from favoring fast shifting stimuli with rewards to slow, changing stimuli that require maintained engagement. In general, little emphasis has been placed on understanding the energy requirement for cognitive performance and on how stimulant medications may affect this. Raichle (2010) has initiated a discussion of this topic and points out that task activation accounts for only a small proportion of the energy expenditure of the brain measured by glucose 
utilization, and that the high-energy expenditure of background activity (in the so-called resting state) likely serves an important purpose. An emerging literature suggests that stimulant drugs decrease the energy requirements to maintain attentional resources engaged, and this could facilitate the engagement of attention for tasks that are energy demanding. Stimulant drugs may also change the cycling of the DMN and the relative predominance of tasknegative (Introspective) over task-positive (Extrospective) brain states. Lapses of attention could reflect either a deficit in ability to maintain deactivation of the DMN or to an overactivation of this network. The analysis of RT distributions and the long reactions may provide a practical way to assess the periodic lapses of attention that are presumed to underlie them.

Despite the marked increase in our understanding of the function of the human brain and the neurobiological substrates underlying ADHD, our approach for treating the cognitive deficits that define this disorder has changed little over the past 50 years. However, one can predict that this will change in the next decade as genetic and epigenetic research identifies new targets for medications, and technologies evolve to help strengthen neuronal circuits that are impaired in ADHD. Similarly, this research may help develop personalized interventions that are tailored to an individual's unique impairments and strengths.

As brain imaging capabilities become more widespread, this may allow imaging studies to be part of clinical characterization for the neurobiological deficits in patients with ADHD and, in the future, these may also help optimize and follow treatment interventions.

\section{DISCLOSURE}

$\mathrm{NV}$ and RB declare that, except for income received from their primary employer, no financial support or compensation has been received from any individual or corporate entity over the past 3 years for research or professional service. JS has received support as an advisor, speaker, and expert witness from pharmaceutical companies (Johnson and Johnson, Shire, and Noven) that manufacture and market stimulant medications for the treatment of ADHD. None of the authors have personal financial holdings that could be perceived as constituting a potential conflict of interest.

\section{REFERENCES}

Abikoff H, Hechtman L, Klein RG, Weiss G, Fleiss K, Etcovitch J et al (2004). Symptomatic improvement in children with ADHD treated with long-term methylphenidate and multimodal psychosocial treatment. J Am Acad Child Adolesc Psychiatry 43: 802-811.

Alderson RM, Rapport MD, Kofler MJ (2007). Attention-deficit/hyperactivity disorder and behavioral inhibition: a meta-analytic review of the stop-signal paradigm. J Abnorm Child Psychol 35: 745-758.

APA (1994). American Psychiatric Association. Diagnostic and Statistical Manual of Mental Disorders, 4th edn (DSM-IV). American Psychiatric Association: Washington, DC.
APA (2000). American Psychiatric Association. Diagnostic and Statistical Manual of Mental Disorders, 4th edn, Text Revisions (DSM-IV-TR). American Psychiatric Association: Washington, DC.

Arnsten A (2009). Toward a new understanding of attention-deficit hyperactivity disorder pathophysiology. CNS Drugs 23: 33-41. A seminal article that modeled the U-shaped interrelationship that exists between arousal state and the release of norepinephrine and dopamine in the prefrontal cortex.

Arnsten A (2006). Stimulants: therapeutic actions in ADHD. Neuropsychopharmacology 31: 2376-2383.

Barkley RA (1997). Behavioral inhibition, sustained attention, and executive functions: constructing a unifying theory of ADHD. Psychol Bull 121: 65-94.

BBC (2010). Editorial standards findings appeals and other editorial issues to the trust considered by the editorial standards committee, 13 January 2010, issued February 2010.

Berridge C, Devilbiss D, Andrzejewski M, Arnsten A, Kelley A, Scheichel B et al (2006). Methylphendiate preferentially increases catecholamine neurotransmission within the prefrontal cortex at low doses that enhance cognitive function. Biol Psychiatry 60: 1111-1120.

Biederman J, Seidman LJ, Petty CR, Fried R, Doyle AE, Cohen DR et al (2008). Effects of stimulant medication on neuropsychological functioning in young adults with attention-deficit/hyperactivity disorder. J Clin Psychiatry 69: 1150-1156.

Bitsakou P, Psychogiou L, Thompson M, Sonuga-Barke EJ (2009). Delay aversion in attention deficit/hyperactivity disorder: an empirical investigation of the broader phenotype. Neuropsychologia 47: 446-456.

Bradley C (1937). The behavior of children receiving Benzedrine. Am J Psychiatry 94: $577-585$

Bush G (2010). Attention-deficit/hyperactivity disorder and attention networks. Neuropsychopharmacology 35: 278-300. A scholarly review of a large literature of brain imaging studies of ADHD, documenting abnormalities in several key brain regions, which pointed at plausible neural mechanisms of ADHD.

Carmona S, Proal E, Hoekzema EA, Gispert JD, Picado M, Moreno I et al (2009). Ventro-striatal reductions underpin symptoms of hyperactivity and impulsivity in attention-deficit/hyperactivity disorder. Biol Psychiatry 66: 972-977.

Casey BJ, Epstein JN, Buhle J, Liston C, Davidson MC, Tonev ST et al (2007). Frontostriatal connectivity and its role in cognitive control in parent-child dyads with ADHD. Am J Psychiatry 164: 1729-1736.

Castellanos FX, Kelly C, Milham MP (2009). The restless brain: attention-deficit hyperactivity disorder, resting-state functional connectivity, and intrasubject variability. Can J Psychiatry 54: 665-672.

Castellanos FX, Lee PP, Sharp W, Jeffries NO, Greenstein DK, Clasen LS et al (2002a). Developmental trajectories of brain volume abnormalities in children and adolescents with attention-deficit/hyperactivity disorder. JAMA 288: 1740-1748.

Castellanos FX, Margulies DS, Kelly C, Uddin LQ, Ghaffari M, Kirsch A et al (2008). Cingulate-precuneus interactions: a new locus of dysfunction in adult attentiondeficit/hyperactivity disorder. Biol Psychiatry 63: 332-337.

Castellanos FX, Sonuga-Barke EJ, Scheres A, Di Martino A, Hyde C, Walters JR (2005). Varieties of attention-deficit/hyperactivity disorder-related intra-individual variability. Biol Psychiatry 57: 1416-1423. FFT analysis was used to compare RTs performance of children with and without ADHD on the Eriksen-Flanker Task, and revealed slow wave oscillations centered at $0.05 \mathrm{~Hz}$ in both groups, but the power at this frequency was $50 \%$ greater for the ADHD than the control group.

Castellanos FX, Tannock R (2002b). Neuroscience of attention-deficit/hyperactivity disorder: the search for endophenotypes. Nat Rev Neurosci 3: 617-628.

Cho SC, Hwang JW, Kim BN, Lee HY, Kim HW, Lee JS et al (2007). The relationship between regional cerebral blood flow and response to methylphenidate in children with attention-deficit hyperactivity disorder: comparison between nonresponders to methylphenidate and responders. J Psychiatr Res 41: 459-465.

Clatworthy PL, Lewis SJ, Brichard L, Hong YT, Izquierdo D, Clark L et al (2009). Dopamine release in dissociable striatal subregions predicts the different effects of oral methylphenidate on reversal learning and spatial working memory. J Neurosci 29: 4690-4696.

Coghill DR, Rhodes SM, Matthews K (2007). The neuropsychological effects of chronic methylphenidate on drug-naive boys with attention-deficit/hyperactivity disorder. Biol Psychiatry 62: 954-962. In a follow-up study of a randomized clinical trial of the acute effects of two doses of methylphenidate relative to placebo, the CANTAB neuropsychological battery was used to evaluate effects of chronic treatment and revealed beneficial effects primarily on tasks not considered to require executive functions.

Conners C (2002). Forty years of methylphenidate treatment in attention-deficit/ hyperactivity disorder. J Atten Disord 6(Suppl 1): S17-S30.

Conners CK, Epstein JN, Angold A, Klaric J (2003). Continuous performance test performance in a normative epidemiological sample. J Abnorm Child Psychol 31: 555-562. 
Corbetta M, Shulman GL (2002). Control of goal-directed and stimulus-driven attention in the brain. Nat Rev Neurosci 3: 201-215.

De Luca M, Beckmann C, De Stefano N, Matthews P, Smith S (2006). fMRI resting state networks define distinct modes of long-distance interactions in the human brain. Neuroimage 29: 1359-1367.

Deminiere JM, Piazza PV, Le Moal M, Simon H (1989). Experimental approach to individual vulnerability to psychostimulant addiction. Neurosci Biobehav Rev 13: 141-147.

Di Martino A, Ghaffari M, Curchack J, Reiss P, Hyde C, Vannucci M et al (2008). Decomposing intra-subject variability in children with attention-deficit/hyperactivity disorder. Biol Psychiatry 64: 607-614.

Diamond DM, Campbell AM, Park CR, Halonen J, Zoladz PR (2007). The temporal dynamics model of emotional memory processing: a synthesis on the neurobiological basis of stress-induced amnesia, flashbulb and traumatic memories, and the Yerkes-Dodson law. Neural Plast 2007: 60803.

Dickstein S, Bannon K, Castellanos FX, MP M (2006). The neural correlates of attention deficit hyperactivity disorder: an ALE meta-analysis. J Child Psychol Psychiatry 47: 1051-1062.

Dodds CM, Muller U, Clark L, van Loon A, Cools R, Robbins TW (2008). Methylphenidate has differential effects on blood oxygenation level-dependent signal related to cognitive subprocesses of reversal learning. J Neurosci 28: 5976-5982.

Dopheide J, Pliszka S (2009). Attention-deficit-hyperactivity disorder: an update. Pharmacotherapy 29: 656-679.

Dosenbach N, Fair D, Miezin FM, Cohen AL, Wenger KK, Dosenbach RA et al (2007). Distinct brain networks for adaptive and stable task control in humans. Proc Natl Acad Sci USA 104: 11073-11078.

Dougherty DD, Bonab AA, Spencer TJ, Rauch SL, Madras BK, Fischman AJ (1999). Dopamine transporter density in patients with attention deficit hyperactivity disorder. Lancet 354: 2132-2133.

Douglas V, Barr R, Desilets J, Sherman E (1995). Do high doses of stimulants impair flexible thinking in attention-deficit hyperactivity disorder? J Am Acad Child Adolesc Psychiatry 34: 877-885.

Drechsler R, Brandeis D, Foldenyi M, Imhof K, Steinhausen HC (2005). The course of neuropsychological functions in children with attention deficit hyperactivity disorder from late childhood to early adolescence. J Child Psychol Psychiatry 46: 824-836.

Durston S, Tottenham NT, Thomas KM, Davidson MC, Eigsti IM, Yang Y et al (2003). Differential patterns of striatal activation in young children with and without ADHD. Biol Psychiatry 53: 871-878. A seminal fMRI study that showed that children with ADHD do not activate frontostriatal regions in the same manner as normally developing children, but rather rely on a more diffuse network of regions, including more posterior and dorsolateral prefrontal regions. This result suggested the recruitment of compensatory mechanisms in the brain of ADHD children.

Epstein JN, Casey BJ, Tonev ST, Davidson MC, Reiss AL, Garrett A et al (2007). ADHD- and medication-related brain activation effects in concordantly affected parent-child dyads with ADHD. J Child Psychol Psychiatry 48: 899-913.

Epstein JN, Conners CK, Hervey AS, Tonev ST, Arnold LE, Abikoff HB et al (2006). Assessing medication effects in the MTA study using neuropsychological outcomes. J Child Psychol Psychiatry 47: 446-456.

Epstein JN, Erkanli A, Conners CK, Klaric J, Costello JE, Angold A (2003). Relations between continuous performance test performance measures and ADHD behaviors. J Abnorm Child Psychol 31: 543-554. A very large epidemiological study of school-aged children, in which the ADHD and control groups did not differ significantly on reaction time (430 vs $394 \mathrm{~ms}$ ), but did on other measures such as RT variability, omission, and commission of errors.

Fan J, McCandliss BD, Sommer T, Raz A, Posner MI (2002). Testing the efficiency and independence of attentional networks. J Cogn Neurosci 14: 340-347.

Fassbender C, Schweitzer J (2006). Is there evidence for neural compensation in attention deficit hyperactivity disorder? A review of the functional neuroimaging literature. Clin Psychol Rev 26: 445-465.

Fox M, Raichle M (2007). Spontaneous fluctuations in brain activity observed with functional magnetic resonance imaging. Nat Rev Neurosci 8: 700-711.

Fox MD, Corbetta M, Snyder AZ, Vincent JL, Raichle ME (2006a). Spontaneous neuronal activity distinguishes human dorsal and ventral attention systems. Proc Natl Acad Sci USA 103: 10046-10051.

Fox MD, Snyder AZ, Zacks JM, Raichle ME (2006b). Coherent spontaneous activity accounts for trial-to-trial variability in human evoked brain responses. Nat Neurosci 9: 23-25.

Fox MD, Snyder AZ, Vincent JK, Corbetta M, Van Essen DC, Raichle ME (2005). The human brain is intrinsically organized into dynamic, anticorrelated functional networks. Proc Natl Acad Sci USA 102: 9673-9678.

Fox M, Zhang D, Snyder A, Raichle M (2009). The global signal and observed anticorrelated resting state brain networks. J Neurophysiol 101: 3270-3283.
Fransson P (2005). Spontaneous low-frequency BOLD signal fluctuations: an fMRI investigation of the resting-state default mode of brain function hypothesis. Hum Brain Mapping 26: 15-29.

Fransson P (2006). How default is the default mode of brain function? Further evidence from intrinsic BOLD signal fluctuations. Neuropsychologia 44: 2836-2845.

Fuster J (1980). The Prefrontal Cortex. University of California: Los Angeles, CA.

Gevensleben H, Holl B, Albrecht B, Vogel C, Schlamp D, Kratz O et al (2009). Is neurofeedback an efficacious treatment for ADHD? A randomised controlled clinical trial. J Child Psychol Psychiatry 50: 780-789.

Giedd JN, Blumenthal J, Molloy E, Castellanos FX (2001). Brain imaging of attention deficit/hyperactivity disorder. Ann NY Acad Sci 931: 33-49.

Groom M, Scerif G, Liddle P, Batty M, Liddle E, Roberts K et al (2010). Effects of motivation and medication on electrophysiological markers of response inhibition in children with attention-deficit/hyperactivity disorder. Biol Psychiatry 67: 624-631.

Haenlein M, Caul WF (1987). Attention deficit disorder with hyperactivity: a specific hypothesis of reward dysfunction. J Am Acad Child Adolesc Psychiatry 26: 356-362.

Halperin JM, Schulz KP (2006). Revisiting the role of the prefrontal cortex in the pathophysiology of attention-deficit/hyperactivity disorder. Psychol Bull 132: 560-581. While most neural models for ADHD pathophysiology have focused on the prefrontal cortex and its interconnections with subcortical structures (e.g., striatum), this important paper puts forth a neurodevelopmental hypothesis according to which ADHD reflects a noncortical dysfunction that manifests early in ontogeny, remains static throughout the lifetime, and is not associated with the remission of symptomatology.

Halperin JM, Trampush JW, Miller CJ, Marks DJ, Newcorn JH (2008). Neuropsychological outcome in adolescents/young adults with childhood ADHD: profiles of persisters, remitters and controls. J Child Psychol Psychiatry 49: 958-966.

Hervey AS, Epstein JN, Curry JF, Tonev S, Eugene Arnold L, Keith Conners C et al (2006). Reaction time distribution analysis of neuropsychological performance in an ADHD sample. Child Neuropsychol 12: 125-140.

Homack S, Riccio CA (2004). A meta-analysis of the sensitivity and specificity of the Stroop Color and Word Test with children. Arch Clin Neuropsychol 19: 725-743.

Hooks MS, Jones GH, Smith AD, Neill DB, Justice Jr JB (1991). Response to novelty predicts the locomotor and nucleus accumbens dopamine response to cocaine. Synapse 9: 121-128.

Huang-Pollock CL, Nigg JT (2003). Searching for the attention deficit in attention deficit hyperactivity disorder: the case of visuospatial orienting. Clin Psychol Rev 23: $801-830$.

laboni F, Douglas VI, Ditto B (1997). Psychophysiological response of ADHD children to reward and extinction. Psychophysiology 34: 116-123.

Jezierski G, Zehle S, Bock J, Braun K, Gruss M (2007). Early stress and chronic methylphenidate cross-sensitize dopaminergic responses in the adolescent medial prefrontal cortex and nucleus accumbens. J Neurochem 103: 2234-2244.

Jin-Shei L, Grshon R, Blitz D, Magasi S, Nowinski C, Reuben D et al (2010). The $\mathrm{NIH}$ Toolbox: a new assessment tool for measuring neurological and behavioral functions. (http://www.nihtoolbox.org/Presentations/Forms/Allltems.aspx).

Johansen EB, Aase H, Meyer A, Sagvolden T (2002). Attention-deficit/hyperactivity disorder (ADHD) behaviour explained by dysfunctioning reinforcement and extinction processes. Behav Brain Res 130: 37-45.

Johansen EB, Killeen PR, Russell VA, Tripp G, Wickens JR, Tannock R et al (2009). Origins of altered reinforcement effects in ADHD. Behav Brain Funct 5: 7.

Johnson KA, Barry E, Bellgrove MA, Cox M, Kelly SP, Daibhis A et al (2008). Dissociation in response to methylphenidate on response variability in a group of medication naive children with ADHD. Neuropsychologia 46: 1532-1541. Results of an attention network task provide evidence of executive function deficits in children diagnosed with ADHD, which cannot be explained by impulsive responding

Jucaite A, Fernell E, Halldin C, Forssberg H, Farde L (2005). Reduced midbrain dopamine transporter binding in male adolescents with attention-deficit/ hyperactivity disorder: association between striatal dopamine markers and motor hyperactivity. Biol Psychiatry 57: 229-238.

Keeler JD, Pichler EE, Ross J (1989). Noise in neural networks: thresholds, hysteresis, and neuromodulation of signal-to-noise. Proc Natl Acad Sci USA 86: 1712-1716.

Kelly A, Uddin L, Biswal B, Castellanos F, Milham M (2008). Competition between functional brain networks mediates behavioral variability. Neuroimage 39: 527-537. Active and resting state fMRI scans were used to quantify the negative correlation between the activities in the default mode (DMN) and task mode (TMN) brain networks. The magnitude of the correlation was found to be related to the intraindividual variability (IIV) of the RT measures of performance on the task. 
Kenemans JL, Bekker EM, Lijffijt M, Overtoom CC, Jonkman LM, Verbaten MN (2005). Attention deficit and impulsivity: selecting, shifting, and stopping. Int J Psychophysiol 58: 59-70.

Kiyatkin EA (2002). Dopamine in the nucleus accumbens: cellular actions, drugand behavior-associated fluctuations, and a possible role in an organism's adaptive activity. Behav Brain Res 137: 27-46.

Kiyatkin EA, Rebec GV (1996). Dopaminergic modulation of glutamate-induced excitations of neurons in the neostriatum and nucleus accumbens of awake, unrestrained rats. J Neurophysiol 75: 142-153.

Klingberg T, Fernell E, Olesen PJ, Johnson M, Gustafsson P, Dahlstrom K et al (2005). Computerized training of working memory in children with ADHD-a randomized, controlled trial. J Am Acad Child Adolesc Psychiatry 44: 177-186.

Knights R (1974). Psychometric assessment of stimulant-induced behavior change. I. In: Conners CE (ed). Clincial Use of Stimulant Drugs in Children. Excerpta Medica: Amsterdam, pp 221-231.

Koepp MJ, Gunn RN, Lawrence AD, Cunningham VJ, Dagher A, Jones T et al (1998). Evidence for striatal dopamine release during a video game. Nature 393 266-268.

Konrad K, Neufang S, Hanisch C, Fink GR, Herpertz-Dahlmann B (2006). Dysfunctional attentional networks in children with attention deficit/hyperactivity disorder: evidence from an event-related functional magnetic resonance imaging study. Biol Psychiatry 59: 643-651. A modified version of the ANT, combined with $\mathrm{FMRI}$, in children with and without ADHD, revealed a complex pattern of group differences, which suggest different strategies for performing the task, with the control group showing the expected activation of the frontalstriatal circuitry, but the ADHD group showing compensatory activation of different brain regions.

Krain A, Castellanos F (2006). Brain development and ADHD. Clin Psychol Rev 26: 433-444.

Krause J (2008). SPECT and PET of the dopamine transporter in attention-deficit/ hyperactivity disorder. Expert Rev Neurother 8: 611-625.

Kuczenski R, Segal DS (1997). An escalating dose/multiple high-dose binge pattern of amphetamine administration results in differential changes in the extracellular dopamine response profiles in caudate-putamen and nucleus accumbens. J Neurosci 17: 4441-4447.

Kuntsi J, Wood AC, Van Der Meere J, Asherson P (2009). Why cognitive performance in ADHD may not reveal true potential: findings from a large population-based sample. J Int Neuropsychol Soc 15: 570-579.

la Fougere C, Krause J, Krause KH, Josef Gildehaus F, Hacker M, Koch W et al (2006). Value of $99 \mathrm{mTc}-T R O D A T-1$ SPECT to predict clinical response to methylphenidate treatment in adults with attention deficit hyperactivity disorder Nucl Med Commun 27: 733-737.

Larisch R, Sitte W, Antke C, Nikolaus S, Franz M, Tress W et al (2006). Striatal dopamine transporter density in drug naive patients with attention-deficit/ hyperactivity disorder. Nucl Med Commun 27: 267-270.

Lehmann K, Lesting J, Polascheck D, Teuchert-Noodt G (2003). Serotonin fibre densities in subcortical areas: differential effects of isolated rearing and methamphetamine. Brain Res Dev Brain Res 147: 143-152.

Lenroot RK, Gledd JN (2008). The changing impact of genes and environment on brain development during childhood and adolescence: initial findings from a neuroimaging study of pediatric twins. Dev Psychopathol 20: 1161-1175.

Leth-Steensen C, Elbaz ZK, Douglas VI (2000). Mean response times, variability, and skew in the responding of ADHD children: a response time distributional approach. Acta Psychol (Amst) 104: 167-190. The variability of performance of ADHD children in a spatial choice RT task differed from control children, and an innovative distribution analysis suggested this increased variability may be attributed to lapses of attention associated with a self-regulation deficit.

Levy $F$ (1991). The dopamine theory of attention deficit hyperactivity disorder (ADHD). Aust N Z J Psychiatry 25: 277-283.

Lijffijt M, Kenemans JL, Verbaten MN, van Engeland H (2005). A meta-analytic review of stopping performance in attention-deficit/hyperactivity disorder: deficient inhibitory motor control? J Abnorm Psychol 114: 216-222.

Luman M, Oosterlaan J, Sergeant JA (2005). The impact of reinforcement contingencies on AD/HD: a review and theoretical appraisal. Clin Psychol Rev 25: 183-213. These authors performed a comprehensive meta-analysis of 22 studies of ADHD and reinforcement contingencies and discuss the implications of their findings, as well as emerging discrepancies, in the context of five different theoretical frameworks used to model ADHD

Marsteller DA, Gerasimov MR, Schiffer WK, Geiger JM, Barnett CR, Schaich Borg J et al (2002). Acute handling stress modulates methylphenidate-induced catecholamine overflow in the medial prefrontal cortex. Neuropsychopharmacology 27: 163-170.

Mehta MA, Owen AM, Sahakian BJ, Mavaddat N, Pickard JD, Robbins TW (2000). Methylphenidate enhances working memory by modulating discrete frontal and parietal lobe regions in the human brain. J Neurosci 20: RC65.
Molina BS, Flory K, Hinshaw SP, Greiner AR, Arnold LE, Swanson JM et al (2007). Delinquent behavior and emerging substance use in the MTA at 36 months: prevalence, course, and treatment effects. J Am Acad Child Adolesc Psychiatry 46: 1028-1040.

Molina BS, Hinshaw SP, Swanson JM, Arnold LE, Vitiello B, Jensen PS et al (2009). The MTA at 8 years: prospective follow-up of children treated for combined-type ADHD in a multisite study. J Am Acad Child Adolesc Psychiatry 48: 484-500. Results of a long-term follow-up of the Multimodal Treatment study of ADHD (MTA) showed no evidence that childhood stimulant medication would have a large protective effect on the emergence of substance use and delinquency, but suggested a slight protective effect of a non-stimulant treatment.

Moll GH, Hause S, Ruther E, Rothenberger A, Huether G (2001). Early methylphenidate administration to young rats causes a persistent reduction in the density of striatal dopamine transporters. J Child Adolesc Psychopharmacol 11: 15-24.

Morcom AM, Fletcher PC (2007). Does the brain have a baseline? Why we should be resisting a rest. Neuroimage 37: 1073-1082.

MTA (1999a). A 14-month randomized clinical trial of treatment strategies for attention-deficit/hyperactivity disorder. The MTA Cooperative Group. Multimodal Treatment Study of Children with ADHD. Arch Gen Psychiatry 56: 1073-1086. Medication management was significantly superior to behavioral modification for the reduction of ADHD symptom - severity and relative benefits on some of the non-symptom-based outcome measures, including performance on reading and math achievement tests.

MTA (1999b). A 14-month randomized clinical trial of treatment strategies for attention-deficit/hyperactivity disorder. The MTA Cooperative Group. Multimodal Treatment Study of Children with ADHD. Arch Gen Psychiatry 56: 1073-1086.

Murphy K, Birn RM, Handwerker DA, Jones TB, Bandettini PA (2009). The impact of global signal regression on resting state correlations: are anti-correlated networks introduced? Neuroimage 44: 893-905.

Nigg JT (2005). Neuropsychologic theory and findings in attention-deficit/ hyperactivity disorder: the state of the field and salient challenges for the coming decade. Biol Psychiatry 57: 1424-1435. This comprehensive review concluded that children with ADHD exhibit many neuropsychological deficits, but none of these alone would be sufficient to characterize the disorder.

Oosterlaan J, Logan GD, Sergeant JA (1998). Response inhibition in AD/HD, CD, comorbid $\mathrm{AD} / \mathrm{HD}+\mathrm{CD}$, anxious, and control children: a meta-analysis of studies with the stop task. J Child Psychol Psychiatry 39: 411-425.

Oosterlaan J, Scheres A, Sergeant JA (2005). Which executive functioning deficits are associated with $\mathrm{AD} / \mathrm{HD}, \mathrm{ODD} / \mathrm{CD}$ and comorbid $\mathrm{AD} / \mathrm{HD}+\mathrm{ODD} / \mathrm{CD}$ ? J Abnorm Child Psychol 33: 69-85.

Ottenbacher K, Cooper H (1983). Drug treatment of hyperactivity in children. Dev Med Child Neurol 25: 358-366.

Paloyelis Y, Mehta MA, Kuntsi J, Asherson P (2007). Functional MRI in ADHD: a systematic literature review. Expert Rev Neurother 7: 1337-1356.

Pennington BF, Ozonoff S (1996). Executive functions and developmental psychopathology. J Child Psychol Psychiatry 37: 51-87.

Peterson BS, Potenza MN, Wang Z, Zhu H, Martin A, Marsh R et al (2009). An FMRI study of the effects of psychostimulants on default-mode processing during Stroop task performance in youths with ADHD. Am J Psychiatry 166: 1286-1294.

Pietrzak RH, Mollica CM, Maruff P, Snyder PJ (2006). Cognitive effects of immediate-release methylphenidate in children with attention-deficit/hyperactivity disorder. Neurosci Biobehav Rev 30: 1225-1245.

Plichta MM, Vasic N, Wolf RC, Lesch KP, Brummer D, Jacob C et al (2009). Neural hyporesponsiveness and hyperresponsiveness during immediate and delayed reward processing in adult attention-deficit/hyperactivity disorder. Biol Psychiatry 65: 7-14

Pliszka SR, Glahn DC, Semrud-Clikeman M, Franklin C, Perez III R, Xiong J et al (2006). Neuroimaging of inhibitory control areas in children with attention deficit hyperactivity disorder who were treatment naive or in long-term treatment. $A m ~ J$ Psychiatry 163: 1052-1060.

Posner MI, Early TS, Reiman E, Pardo PJ, Dhawan M (1988). Asymmetries in hemispheric control of attention in schizophrenia. Arch Gen Psychiatry 45 814-821.

Posner Ml, Petersen SE (1990). The attention system of the human brain. Annu Rev Neurosci 13: 25-42.

Posner Ml, Rothbart MK (2007). Research on attention networks as a model for the integration of psychological science. Annu Rev Psychol 58: 1-23.

Posner MI, Rothbart MK (2009). Toward a physical basis of attention and self regulation. Phys Life Rev 6: 103-120. This review introduces the importance of understanding the physical bases of self regulation for improving our understanding of individual differences in brain function that could lead or accompany pathological states such as ADHD. 
Powers RL, Marks DJ, Miller CJ, Newcorn JH, Halperin JM (2008). Stimulant treatment in children with attention-deficit/hyperactivity disorder moderates adolescent academic outcome. J Child Adolesc Psychopharmacol 18: 449-459.

Raichle M (2010). Two views of brain function. Trends Cogn Sci 14: 180-190. A review that highlights the importance of exploring intrinsic activity to fully understand integration of neural activity, an area of brain research that has been relatively ignored in traditional studies of brain function.

Rapoport J, Buchsbaum M, Zahn T, Weingartner H, Ludlow C, Mikkelsen E (1978). Dextroamphetamine: cognitive and behavioral effects in normal prepubertal boys. Science 199: 560-563.

Rapoport JL, Gogtay N (2008). Brain neuroplasticity in healthy, hyperactive and psychotic children: insights from neuroimaging. Neuropsychopharmacology 33: 181-197.

Rapport MD, Kelley A (1993). Psychostimulant effects on learning and cognitive function. In: Matson $\mathrm{JL}(\mathrm{ed})$. Handbook of Hyperactivity in Children. Allyn \& Bacon: Boston, MA. pp. 97-136.

Rhodes SM, Coghill DR, Matthews K (2005). Neuropsychological functioning in stimulant-naive boys with hyperkinetic disorder. Psychol Med 35: 1109-1120.

Rhodes SM, Coghill DR, Matthews K (2006). Acute neuropsychological effects of methylphenidate in stimulant drug-naive boys with ADHD II - broader executive and non-executive domains. J Child Psychol Psychiatry 47: 1184-1194. Application of the CANTAB battery to children with ADHD detected significant group differences not only for domains related to executive or frontal lobe function (working memory, planning and strategy formation, and set-shifting), but also for domains related to temporal and parietal lobe function (spatial recognition and span, pattern recognition, and delayed matching to sample.

Robbins T, Sahakian B (1979). Paradoxical effects of psychomotor stimulant drugs in hyperactive children from the standpoint of behavioural pharmacology. Neuropharmacology 18: 931-950.

Robbins TW, Arnsten AF (2009). The neuropsychopharmacology of fronto-executive function: monoaminergic modulation. Annu Rev Neurosci 32: 267-287.

Rolls ET, Thorpe SJ, Boytim M, Szabo I, Perrett DI (1984). Responses of striatal neurons in the behaving monkey. 3. Effects of iontophoretically applied dopamine on normal responsiveness. Neuroscience 12: 1201-1212.

Rubia K, Halari R, Cubillo A, Mohammad AM, Brammer M, Taylor E (2009). Methylphenidate normalises activation and functional connectivity deficits in attention and motivation networks in medication-naive children with ADHD during a rewarded continuous performance task. Neuropharmacology 57: 640-652.

Rubia K, Smith A, Taylor E (2007). Performance of children with attention deficit hyperactivity disorder (ADHD) on a test battery of impulsiveness. Child Neuropsychol 13: 276-304. The Maudsley attention and response suppression battery uncovered specific deficits among children with ADHD on the tests of motor inhibition, sustained attention, and time discrimination, but not on a test of cognitive inhibition.

Sahakian B, Morein-Zamir S (2007). Professor's little helper. Nature 450: 1157-1159.

Sahakian B, Robbins T (1977). Are the effects of psychomotor stimulant drugs in hyperactive children really paradoxical? Med Hypotheses 3: 154-158.

Scheres A, Oosterlaan J, Geurts H, Morein-Zamir S, Meiran N, Schut H et al (2004). Executive functioning in boys with ADHD: primarily an inhibition deficit? Arch Clin Neuropsychol 19: 569-594.

Scheres A, Oosterlaan J, Swanson J, Morein-Zamir S, Meiran N, Schut H et al (2003). The effect of methylphenidate on three forms of response inhibition in boys with AD/HD. J Abnorm Child Psychol 31: 105-120.

Schulz KP, Fan J, Tang CY, Newcorn JH, Buchsbaum MS, Cheung AM et al (2004). Response inhibition in adolescents diagnosed with attention deficit hyperactivity disorder during childhood: an event-related FMRI study. Am J Psychiatry 161: 1650-1657.

Schweitzer JB, Lee DO, Hanford RB, Zink CF, Ely TD, Tagamets MA et al (2004). Effect of methylphenidate on executive functioning in adults with attentiondeficit/hyperactivity disorder: normalization of behavior but not related brain activity. Biol Psychiatry 56: 597-606.

Seamans JK, Yang CR (2004). The principal features and mechanisms of dopamine modulation in the prefrontal cortex. Prog Neurobiol 74: 1-58.

Sergeant J, Scholten C (1983). A stages-of-information approach to hyperactivity. J Child Psychol Psychiatry 24: 49-60.

Sergeant JA, Banaschewski T, Sonuga-barke E, Zuddas A (2009). 20th EUNETHYDIS Meeting: Current Controversies and Emerging Themes, Winchester, UK, 30 September-3 October 2009.

Seu E, Lang A, Rivera RJ, Jentsch JD (2009). Inhibition of the norepinephrine transporter improves behavioral flexibility in rats and monkeys. Psychopharmacology (Berl) 202: 505-519.
Shanahan MA, Pennington BF, Willcutt EW (2008). Do motivational incentives reduce the inhibition deficit in ADHD? Dev Neuropsychol 33: 137-159.

Shaw P, Gogtay N, Rapoport J (2010). Childhood psychiatric disorders as anomalies in neurodevelopmental trajectories. Hum Brain Mapp 31: 917-925.

Shaw P, Gornick M, Lerch J, Addington A, Seal J, Greenstein D et al (2007). Polymorphisms of the dopamine D4 receptor, clinical outcome, and cortical structure in attention-deficit/hyperactivity disorder. Arch Gen Psychiatry 64: 921-931.

Shaw P, Lerch J, Greenstein D, Sharp W, Clasen L, Evans A et al (2006). Longitudinal mapping of cortical thickness and clinical outcome in children and adolescents with attention-deficit/hyperactivity disorder. Arch Gen Psychiatry 63: 540-549.

Shiels K, Hawk Jr LW, Lysczek CL, Tannock R, Pelham Jr WE, Spencer SV et al (2008). The effects of incentives on visual-spatial working memory in children with attention-deficit/hyperactivity disorder. J Abnorm Child Psychol 36: 903-913.

Solanto MV (1998). Neuropsychopharmacological mechanisms of stimulant drug action in attention-deficit hyperactivity disorder: a review and integration. Behav Brain Res 94: 127-152.

Sonuga-Barke E, Castellanos F (2007). Spontaneous attentional fluctuations in impaired states and pathological conditions: a neurobiological hypothesis. Neurosci Biobehav Rev 31: 977-986. This influential review proposed an alternative hypothesis to the etiology of ADHD, in which spontaneous patterns of very low frequency $(<0.1 \mathrm{~Hz})$ coherence within the so called brain default (active during rest) network, can persist or intrude into periods of active task-specific processing, producing periodic fluctuations in attention that compete with goal-directed activity.

Sonuga-Barke EJ (2002). Psychological heterogeneity in AD/HD — a dual pathway model of behaviour and cognition. Behav Brain Res 130: 29-36.

Spencer SV, Hawk Jr LW, Richards JB, Shiels K, Pelham Jr WE, Waxmonsky JG (2009). Stimulant treatment reduces lapses in attention among children with ADHD: the effects of methylphenidate on intra-individual response time distributions. J Abnorm Child Psychol 37: 805-816.

Spencer TJ, Biederman J, Madras BK, Dougherty DD, Bonab AA, Livni E et al (2007). Further evidence of dopamine transporter dysregulation in ADHD: a controlled PET imaging study using altropane. Biol Psychiatry 62: 1059-1061.

Sprague RL, Sleator EK (1977). Methylphenidate in hyperkinetic children: differences in dose effects on learning and social behavior. Science 198: 1274-1276.

Stix G (2009). Turbocharging the brain. Sci Am 301: 46-49, 52-55.

Strohle A, Stoy M, Wrase J, Schwarzer S, Schlagenhauf F, Huss M et al (2008). Reward anticipation and outcomes in adult males with attention-deficit/ hyperactivity disorder. Neuroimage 39: 966-972.

Swanson J, Arnold LE, Kraemer H, Hechtman L, Molina B, Hinshaw S et al (2008a). Evidence, interpretation, and qualification from multiple reports of long-term outcomes in the Multimodal Treatment study of Children with ADHD (MTA): part I: executive summary. J Atten Disord 12: 4-14.

Swanson J, Arnold LE, Kraemer H, Hechtman L, Molina B, Hinshaw S et al (2008b). Evidence, interpretation, and qualification from multiple reports of long-term outcomes in the Multimodal Treatment Study of children with ADHD (MTA): part II: supporting details. J Atten Disord 12: 15-43.

Swanson J, Cantwell D, Lerner M, McBurnett K, Hanna G (1991a). Effects of stimulant medication on learning in children with ADHD. J Learn Disabil 24: 219-230.

Swanson J, Gupta S, Guinta D, Flynn D, Agler D, Lerner M et al (1999). Acute tolerance to methylphenidate in the treatment of attention deficit hyperactivity disorder in children. Clin Pharmacol Ther 66: 295-305.

Swanson J, Wigal T, Lakes K, Volkow N (in press). Attention deficit hyperactivity disorder: defining a spectrum disorder. Illes $\mathrm{J}$ and Sahakian BJ (eds). Oxford Handbook of Neuroethics. Oxford University Press: Oxford, UK.

Swanson JM, Posner M, Potkin S, Bonforte S, Youpa D, Fiore C et al (1991b). Activating tasks for the study of visual-spatial attention in ADHD children: a cognitive anatomic approach. J Child Neurol 6(Suppl): S119-S127.

Tamm L, Menon V, Ringel J, Reiss AL (2004). Event-related FMRI evidence of frontotemporal involvement in aberrant response inhibition and task switching in attention-deficit/hyperactivity disorder. J Am Acad Child Adolesc Psychiatry 43: 1430-1440. On a Go-NoGo task, an ADHD group made more errors than the control group, which produced increased activation of the posterior cingulate and dorsolateral prefrontal and parietal brain regions attributed to compensatory processes used to perform this inhibition task.

Tian L, Jiang T, Wang Y, Zang Y, He Y, Liang M et al (2006). Altered resting-state functional connectivity patterns of anterior cingulate cortex in adolescents with attention deficit hyperactivity disorder. Neurosci Lett 400: 39-43.

Tomasi D, Volkow N, Wang R, Telang F, Wang G, Chang L et al (2009). Dopamine transporters in striatum correlate with deactivation in the default mode network during visuospatial attention. PLoS One 4: e6102. 
Tripp G, Wickens J (2009). Reinforcement and ADHD Workshop. Okinawa Institute of Science and Technology, 8-10 September 2009, Okinawa, Japan.

Tripp G, Wickens JR (2008). Research review: dopamine transfer deficit: a neurobiological theory of altered reinforcement mechanisms in ADHD. J Child Psychol Psychiatry 49: 691-704.

Uddin LQ, Kelly AM, Biswal BB, Margulies DS, Shehzad Z, Shaw D et al (2008). Network homogeneity reveals decreased integrity of default-mode network in ADHD. J Neurosci Methods 169: 249-254.

Udo de Haes Jl, Maguire RP, Jager PL, Paans AM, den Boer JA (2007). Methylphenidate-induced activation of the anterior cingulate but not the striatum: a $\left[{ }^{15} \mathrm{O}\right] \mathrm{H}_{2} \mathrm{O}$ PET study in healthy volunteers. Hum Brain Mapp 28: 625-635.

Vaidya C, Austin G, Kirkorian G, Ridlehuber H, Desmond J, Glover G et al (1998). Selective effects of methylphenidate in attention deficit hyperactivity disorder: a functional magnetic resonance study. Proc Natl Acad Sci USA 95 14494-14499.

Van Dijk KR, Hedden T, Venkataraman A, Evans KC, Lazar SW, Buckner RL (2009). Intrinsic functional connectivity as a tool for human connectomics: theory, properties, and optimization. J Neurophysiol 103: 297-321.

van Mourik R, Oosterlaan J, Sergeant JA (2005). The Stroop revisited: a metaanalysis of interference control in AD/HD. J Child Psychol Psychiatry 46: 150-165.

Vaurio RG, Simmonds DJ, Mostofsky SH (2009). Increased intra-individual reaction time variability in attention-deficit/hyperactivity disorder across response inhibition tasks with different cognitive demands. Neuropsychologia 47: 2389-2396.

Villemagne VL, Wong DF, Yokoi F, Stephane M, Rice KC, Matecka D et al (1999). GBR12909 attenuates amphetamine-induced striatal dopamine release as measured by $[(11) C]$ raclopride continuous infusion PET scans. Synapse 33 268-273.

Vitiello B, Severe JB, Greenhill LL, Arnold LE, Abikoff HB, Bukstein OG et al (2001). Methylphenidate dosage for children with ADHD over time under controlled conditions: lessons from the MTA. J Am Acad Child Adolesc Psychiatry 40: 188-196.

Volkow N, Wang G, Fowler J, Gatley S, Logan J, Ding Y et al (1998). Dopamine transporter occupancies in the human brain induced by therapeutic doses of oral methylphenidate. Am J Psychiatry 155: 1325-1331.

Volkow N, Wang G, Fowler JS, Telang F, Maynard L, Logan J et al (2004). Evidence that methylphenidate enhances the saliency of a mathematical task by increasing dopamine in the human brain. Am J Psychiatry 161: 1173-1180.

Volkow N, Wang GJ, Newcorn J, Fowler JS, Telang F, Solanto MV et al (2007). Brain dopamine transporter levels in treatment and drug naïve adults with ADHD. Neuroimage 34: 1182-1190.

Volkow ND, Ding YS, Fowler JS, Wang GJ, Logan J, Gatley JS et al (1995). Is methylphenidate like cocaine? Studies on their pharmacokinetics and distribution in the human brain. Arch Gen Psychiatry 52: 456-463. In this seminal review the authors speculate that the slow clearance of methylphenidate from the brain may serve as a limiting factor in promoting its frequent self-administration.

Volkow ND, Fowler JS, Logan J, Alexoff D, Zhu W, Telang F et al (2009a). Effects of modafinil on dopamine and dopamine transporters in the male human brain: clinical implications. JAMA 301: 1148-1154.

Volkow ND, Fowler JS, Wang GJ, Telang F, Logan J, Wong C et al (2008). Methylphenidate decreased the amount of glucose needed by the brain to perform a cognitive task. PLoS One 3: e2017.

Volkow ND, Wang G, Fowler JS, Logan J, Gerasimov M, Maynard L et al (2001). Therapeutic doses of oral methylphenidate significantly increase extracellular dopamine in the human brain. J Neurosci 21: RC121.
Volkow ND, Wang GJ, Fowler JS, Gatley SJ, Logan J, Ding YS et al (1999a) Blockade of striatal dopamine transporters by intravenous methylphenidate is not sufficient to induce self-reports of 'high'. J Pharmacol Exp Ther 288: 14-20.

Volkow ND, Wang GJ, Fowler JS, Logan J, Franceschi D, Maynard L et al (2002). Relationship between blockade of dopamine transporters by oral methylphenidate and the increases in extracellular dopamine: therapeutic implications. Synapse 43: 181-187.

Volkow ND, Wang GJ, Fowler JS, Logan J, Gatley SJ, Wong C et al (1999b). Reinforcing effects of psychostimulants in humans are associated with increases in brain dopamine and occupancy of D(2) receptors. J Pharmacol Exp Ther 291 409-415

Volkow ND, Wang GJ, Fowler JS, Logan J, Jayne M, Franceschi D et al (2002). 'Nonhedonic' food motivation in humans involves dopamine in the dorsal striatum and methylphenidate amplifies this effect. Synapse 44: 175-180.

Volkow ND, Wang GJ, Kollins SH, Wigal TL, Newcorn JH, Telang F et al (2009b). Evaluating dopamine reward pathway in ADHD: clinical implications. JAMA 302 1084-1091. This seminal paper reported for the first time that the dopamine reward pathway of subjects with ADHD display a reduction in dopamine synaptic markers that was associated with symptoms of inattention.

Walters J, Ruskin D, Baek D, Allers K, Bergstrom D (2001). Cognitive function paradigms: implications of neurophysiological studies of dopamine stimulants for Tourette syndrome and comorbid attention-deficit hyperactivity disorder. Adv Neurol 85: 133-149.

Wang GJ, Volkow N, Wigal T, Kollins S, Newcorn J, Telang F et al (eds) (2009). Chronic methylphenidate treatment increases striatal dopamine transporter availability in patients with attention deficit hyperactive disorder. American College of Neuropsychopharmacology 48th Annual Meeting, poster no. 186.

Waxmonsky $J$ (2005). Nonstimulant therapies for attention-deficit hyperactivity disorder (ADHD) in children and adults. Essent Psychopharmacol 6: 262-276.

Weissman DH, Roberts KC, Visscher KM, Woldorff MG (2006). The neural bases of momentary lapses in attention. Nat Neurosci 9: 971-978.

Wender PH (1971). Minimal Brain Dysfunction in Children. John Wiley \& Sons Inc: New York.

Wilens TE (2008). Effects of methylphenidate on the catecholaminergic system in attention-deficit/hyperactivity disorder. J Clin Psychopharmacol 28(Suppl 2): S46-S53.

Willcutt EG, Doyle AE, Nigg JT, Faraone SV, Pennington BF (2005). Validity of the executive function theory of attention-deficit/hyperactivity disorder: a metaanalytic review. Biol Psychiatry 57: 1336-1346. A seminal review that found little evidence to support the hypothesis that executive function deficits are necessary and sufficient cause of ADHD

Winstanley CA, Eagle DM, Robbins TW (2006). Behavioral models of impulsivity in relation to ADHD: translation between clinical and preclinical studies. Clin Psychol Rev 26: 379-395.

Wolf R, Plichta MM, Sambataro F, Fallgatter AJ, Jacob C, Lesch KP et al (2009). Regional brain activation changes and abnormal functional connectivity of the ventrolateral prefrontal cortex during working memory processing in adults with attention-deficit/hyperactivity disorder. Hum Brain Mapp 30: 2252-2266.

Woodward DJ, Moises HC, Waterhouse BD, Hoffer BJ, Freedman R (1979). Modulatory actions of norepinephrine in the central nervous system. Fed Proc 38: 2109-2116

Yang H, Li OQ, Zhang MM, Long XY (2010). Spontaneous brain activity in medication-naive ADHD boys revealed by ALFF analysis. Brain Dev (in press).

Zang YF, He Y, Zhu CZ, Cao QJ, Sui MQ, Liang M et al (2007). Altered baseline brain activity in children with ADHD revealed by resting-state functional MRI. Brain Dev 29: 83-91. 